\title{
Inflación venezolana: Variaciones trimestrales nominales y reales de Venezuela en el periodo 2005-2017
}

\author{
Venezuelan inflation. Nominal \\ and real quarterly variations of \\ Venezuela in the 2005-2017 period
}

DOI: https://doi.org/10.17981/econcuc.41.1.2020.Econ.6

Artículo de investigación.

Fecha de recepción: 13/10/2019

Fecha de devolución: 10/01/2020

Fecha de aceptación: 22/01/2020

Fecha de publicación: 27/01/2020

\section{Remedios Pitre Redondo \\ Universidad de la Guajira. La Guajira (Colombia) \\ rpitre@uniguajira.edu.co}

\author{
Armando Urdaneta Montiel \\ Universidad Metropolitana del Ecuador. \\ Guayaquil (Ecuador) \\ aurdaneta@umet.edu.ec

\section{Hugo Hernández Palma \\ Universidad del Atlántico. \\ Barranquilla (Colombia)} \\ hugohernandezp@mail.uniatlantico.edu.co
}

Para citar este artículo:

Pitre, R., Urdaneta, A. \& Hernández, H. (2020). Inflación venezolana: Variaciones trimestrales nominales y reales de Venezuela en el periodo 2005-2017. Económicas CUC, 41(1), 121-142. DOI: https://doi.org/10.17981/econcuc.41.1.2020.Econ.6

\section{Resumen}

Esta investigación estudio el crédito y la inflación venezolana durante el periodo 2005-2017. Para ello se planteó como objetivo el identificar las variaciones trimestrales nominales y reales de Venezuela en el periodo 2005-2017. Metodológicamente se fundamentó desde la investigación cuantitativa, siendo este un estudio correlacional, en el cual los datos fueron tomados de los reportes de información estadística suministrados por el Banco Central de Venezuela en el 2017 y SUDEBAN en el 2017. Los resultados permitieron observar los comportamientos de las variaciones trimestrales nominales y reales de Venezuela, permitiendo considerar la gran relevancia que tienen los procesos inflacionarios en las dinámicas de las naciones tanto a nivel local como a nivel internacional. Destacando como en los índices financieros de la nación venezolana en los datos oficiales se observa una contradicción con la realidad actual.

Palabras clave: Variaciones trimestrales; nominales; reales; inflación; índices financieros

\section{Abstract}

This research studied credit and Venezuelan inflation during the period 2005-2017. The objective was to identify nominal and real quarterly variations in Venezuela during the period 2005-2017. Methodologically it was based on quantitative research, being this a correlational study, in which the data was taken from the statistical information reports provided by the Central Bank of Venezuela in 2017 and SUDEBAN in 2017. The results allowed us to observe the behavior of nominal and real quarterly variations in Venezuela, allowing us to consider the great relevance that inflationary processes have in the dynamics of nations both locally and internationally. Highlighting how in the financial indexes of the Venezuelan nation in the official data there is a contradiction with the current reality.

Keywords: Artificial credit expansion; inflation; monetary liquidity; monetary base; financial indexes 


\section{INTRODUCCIÓN}

Actualmente las dinámicas internacionales requieren que se apliquen las diversas técnicas y análisis en el campo de la práctica tanto a nivel organizacional como a nivel estatal para garantizar el éxito de estas dentro del actual mercado competitivo (Hernández, 2011; Henríquez, Rada y Torrenerga, 2016; Paredes, 2017). Ciertamente, en este contexto se destacan los países caribeños al estar sumamente inmersos en estos procesos de globalización económica (Herrera, Balbis, Gómez y Hernández, 2015; Casas, 2017).

La discusión clásica del papel moneda fue introducida, entre otros, por Thornton (2000), quien diferenció el papel moneda del dinero de pleno contenido, así como los mecanismos que promueven su expansión a través del Banco Central y cómo ese crecimiento promueve la inflación. Así, para Thornton (2000), el incremento en la cantidad de dinero tiende a aumentar el precio de las mercancías y la reducción en su emisión impulsará el precio de las mercancías a la baja.

Las variaciones relativas en el valor de cambio de algunos productos son, según Wicksell (1962), el resultado de cambios en las condiciones de producción de las mejoras técnica-científicas, entre otros factores; del mismo modo se percató que pueden existir cambios en los precios monetarios de todos o la mayoría de los productos sin estar asociados a los factores antes expuestos. Por ello, considera que los incrementos en los precios no significa que se incrementen los precios de todos los productos, ya que algunos, por diferentes razones, permanecerán estables.

Estos problemas en la determinación de los precios a nivel general llevaron a Wicksell (1962) a apoyarse en la teoría de la utilidad marginal y en la determinación del nivel promedio de los precios, que debería seguir la "ley de indiferencia” de Jevons (1998).

Estos importantes aportes de Wicksell (1962) han ayudado a abrir una segunda vertiente de lo que se ha dado por llamar como Teoría Austriaca del Ciclo Económico (Austrian Business Cycle Theory).

Años atrás, estudios sobre el incremento de la cantidad de dinero de Mises (1982) apuntan a que su consecuencia inmediata es la disminución de la utilidad marginal de cada unidad monetaria y una demanda de más bienes por los consumidores. El resultado será un incremento en los precios de los productos deseados, una baja en el valor de cambio objetivo del dinero y el ingreso real de las personas.

Esta última idea vincula el planteamiento de Mises (1982) con el de Cantillón (2002) en el sentido de que todo aumento de la oferta de dinero no se distribuye uniformemente como parece suponer la teoría cuantitativa del dinero. De esta manera, el aumento de la oferta de dinero hace que cierto grupo social experimente un incremento de sus medios de pagos, que los impulsa al mercado a adquirir productos. Los sectores económicos que sientan una mayor presión de demanda tenderán a aumentar sus precios de oferta en comparación con aquellos 
que no experimentan una demanda correspondiente al aumento de la oferta de dinero. El resultado es similar al expuesto por Wicksell (1962) en términos de la tasa de interés y restructuración del aparato productivo.

En ese sentido Gómez (2008), citando a Mises (1982), señala que esa expansión de la oferta de dinero es potenciada por el Banco Central y los intermediarios bancarios con la creación secundaria de dinero (Casas, 2017). Ello se debe fundamentalmente como lo plantea De Soto (2011), a un estiramiento máximo en lo que se conoce como "principio de doble disponibilidad" del "depósito irregular" (Azuero, 1990; Zúñiga, 2017). El esquema de la Figura 1 muestra cómo la expansión del crédito se sustenta en el principio de la disponibilidad que tiene un banco de destinar los fondos prestables de los depositantes.

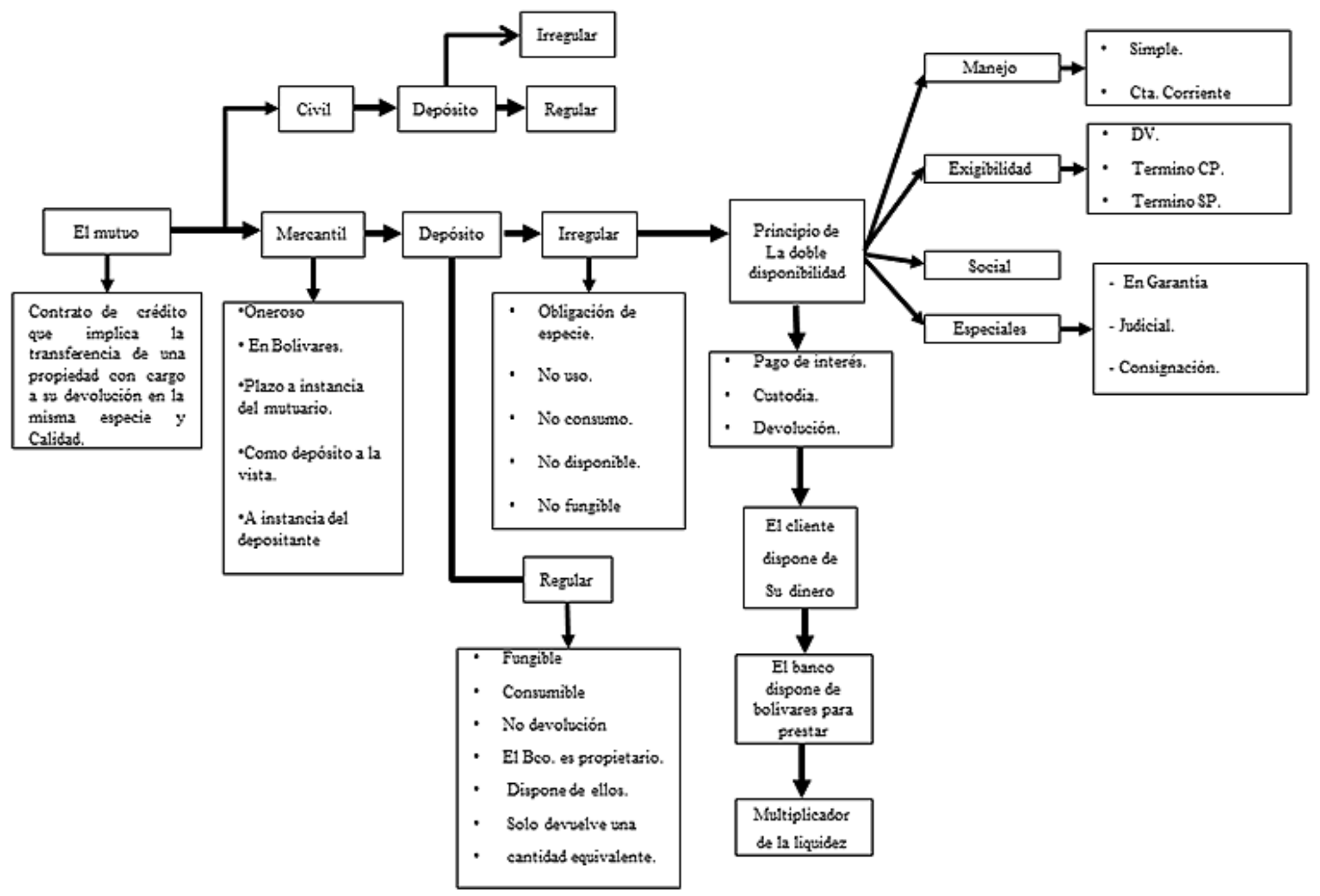

Figura 1. Principio de la doble disponibilidad.

Fuente: Elaboración propia basada en Azuero (1990).

Ahora bien, De Soto (2011) y Zúñiga (2017) aclaran que un sistema bancario con reserva fraccionaria los bancos pueden disponer del 100\% de los depósitos del público menos el porcentaje estipulado por el Banco Central como encaje legal, más el dinero secundario creado por ellos. El esquema de De Soto (2011) puede ser expuesto en la Tabla 1. 
TABLA 1.

Diferencias económicas y legales entre los contratos de préstamos monetarios y los de depósitos irregulares

\begin{tabular}{|c|c|c|}
\hline & Préstanos monetarios & Depósitos monetarios irregulares \\
\hline $\begin{array}{l}\text { Diferencias } \\
\text { económicas }\end{array}$ & $\begin{array}{l}\text { Los bienes presentes son cambiados por } \\
\text { futuros. } \\
\text { El dinero disponible es transferido } \\
\text { desde el prestamista al prestatario. La } \\
\text { propiedad del dinero es transferida. } \\
\text { Los intereses son pagados como bienes } \\
\text { presentes y los bienes futuros son } \\
\text { intercambiados }\end{array}$ & $\begin{array}{l}\text { Los bienes presentes no son } \\
\text { cambiados por bienes futuros. } \\
\text { Existe completa disponibilidad } \\
\text { para el prestamista y no hay } \\
\text { transferencia de la propiedad. } \\
\text { El interés no es pagado con bienes } \\
\text { presentes y los bienes futuros no son } \\
\text { cambiados }\end{array}$ \\
\hline Diferencias legales & $\begin{array}{l}\text { El elemento esencial es la transferencia } \\
\text { de la disponibilidad de los bienes } \\
\text { presentes al prestatario. } \\
\text { El contrato requiere el establecimiento } \\
\text { de un término para el retorno del } \\
\text { principal y los intereses. } \\
\text { La obligación del prestatario es } \\
\text { retornar el tantundem (un monto de } \\
\text { igual cantidad y cualidad pero no } \\
\text { necesariamente las mismas unidades } \\
\text { exactas) al final del término acordado } \\
\text { y el pago acordado en el monto de los } \\
\text { intereses }\end{array}$ & $\begin{array}{l}\text { El elemento esencial es asegurarse } \\
\text { del tantundem. } \\
\text { No existe término, el contrato es } \\
\text { según se demande. } \\
\text { La obligación del prestatario es } \\
\text { mantener el tantundem disponible } \\
\text { todo el tiempo (100\% en reservas) }\end{array}$ \\
\hline
\end{tabular}

Fuente: Elaboración propia basada en De Soto (2011).

En consecuencia, de acuerdo a De Soto (2011), se produciría lo siguiente:

El sistema bancario de reserva fraccional genera una oferta extremadamente elástica de dinero, que se ampliaría con facilidad pero que deberá contraerse esforzadamente, produciendo los correspondientes efectos sobre la actividad económica, que estará repetidamente golpeada por sucesivos procesos de expansiones y recesiones. La "maniacadepresiva" actividad económica con todo su peso de grandes costos sociales, es indudablemente el más severo efecto dañino al sistema bancario existente (basado en reservas fraccionales, en violación de principios legales universales de la sociedad) (De Soto, 2009, p. 261).

Estos proyectos, según Gómez (2008) y Henríquez, Rada y Torrenegra (2016), se hacen muy costosos, y se revelan económicamente insostenibles debido al crecimiento exponencial de los precios, producto, primeramente, de la expansión monetaria y luego a la expansión artificial del crédito; la única manera de recuperar estos proyectos sería un nuevo aumento de la oferta de dinero por decisión del Banco Central. Pero tal decisión repetiría el proceso anterior infinitamente, de manera acumulativa (Wicksell, 1962)

En este sentido, al revisar el caso venezolano se observa como actualmente la crisis económica ha dificultado de manera significativamente la calidad de vida del ciudadano por lo que el presente artículo se realiza con el objetivo de identificar las variaciones trimestrales nominales y reales de Venezuela en el periodo 2005-2017. 


\section{Metodología}

En función a lo antes planteado, la investigación se concibe en esencia como un estudio correlacional, el cual busca comprender las relaciones existentes sobre las variaciones trimestrales nominales y reales de Venezuela en el periodo 2005-2017. Sigue un diseño no experimental (Palella y Martins, 2012), donde no se manipulan las variables involucradas y se analizan los resultados dentro del contexto donde fueron obtenidos los datos.

En cuanto al procedimiento de investigación (Hernández, Fernández y Baptista, 2010), los datos fueron tomados de los reportes de información estadística suministrados por el Banco Central de Venezuela (BCV, 2017) y la Superintendencia de Bancos y Otras Instituciones de Crédito (SUDEBAN, 2017) en lo concerniente a los agregados monetarios e indicadores e índices financieros.

Se trabajó con las series de tiempo de las Variaciones Trimestrales de la Base Monetaria (VTBM), Liquidez Monetaria (VTM1), Cartera Neta de Crédito (VTCCN), el Índice Nacional de Precios al Consumidor (VTINPC) y la Cartera de Crédito Expandida Artificialmente (VTCCNEA), como variables endógenas, empleando Vectores Autorregresivos Estructurados (VARE). A partir de una previa descripción en series de tiempo de las variaciones trimestrales de índices económicos y agregados monetarios a precios corrientes, de los índices e indicadores económicos y agregados monetarios en términos reales.

Seguidamente se procedió a la estimación de vectores autorregresivos y de cointegración para hacer pronóstico de las variaciones trimestrales y determinar su relación a largo plazo. Se realizaron pruebas de raíz unitaria de las variables endógenas para determinar la pertinencia en el uso de VARE, consecutivamente se realizaron el test de causalidad para determinar las variables endógenas de cada VARE, evidenciando las relaciones causales entre ellas para la construcción de los VARE; luego la prueba de rezagos ideales significativos para establecer sí los coeficientes autorregresivos del modelo VARE hacen una estimación explicativa de las variables endógenas dependientes. Además de lo anterior, se ejecutó la prueba de cointegración de Johansen para estimar los vectores de cointegración en su relación a largo plazo, posteriormente se efectuarán las pruebas de autocorrelación a través de los correlogramas, función impulso respuesta y descomposición de la varianza.

Todo esto en aras de estimar los vectores autorregresivos y de cointegración que permitan evaluar la relación de la expansión artificial del crédito con el fenómeno inflacionario en Venezuela en el período 2005-2017, tomando como referencia la Teoría Austriaca del Dinero del Ciclo Económico (Austrian Business Cycle Theory) en la vertiente de Wicksell (Braun, 2013), el elemento crédito abordador por Mises (1981) y el punto de vista de De Soto (2011) acerca de la expansión artificial del crédito, producto de la violación del derecho al depósito irregular por parte del sector bancario. 


\section{RESULTADOS}

Al estimar el coeficiente de correlación de Pearson (Anderson, Sweeney y Willians, 2008), que determina el grado de asociación lineal de dos variables; el cual para este caso sus variaciones pueden evidenciar que tanto VTM1 y VTCCNEA presentan entre ellas un alto coeficiente de correlación lineal de 0,89. Mientras que al estimar dicho coeficiente de las variaciones trimestrales de cada variable con respecto VTINPC sus valores fueron 0,91 para VTM1 y 0,84 para VTCCNEA. Al estimar la covarianza entre M1 (Liquidez Monetaria), INPC (Índice Nacional de Precios al Consumidor), y este con la Cartera de Créditos Neta Expandida Artificialmente (CCNEA), se obtuvo que dichos estadísticos eran Sxy $>0$. La Figura 2 muestra como existe una variación muy similar entre el índice nacional de precios al consumidor de $17,52 \%$, la liquidez monetaria M1 de 20,57\% y la cartera de créditos neta expandida artificialmente de $19,65 \%$.

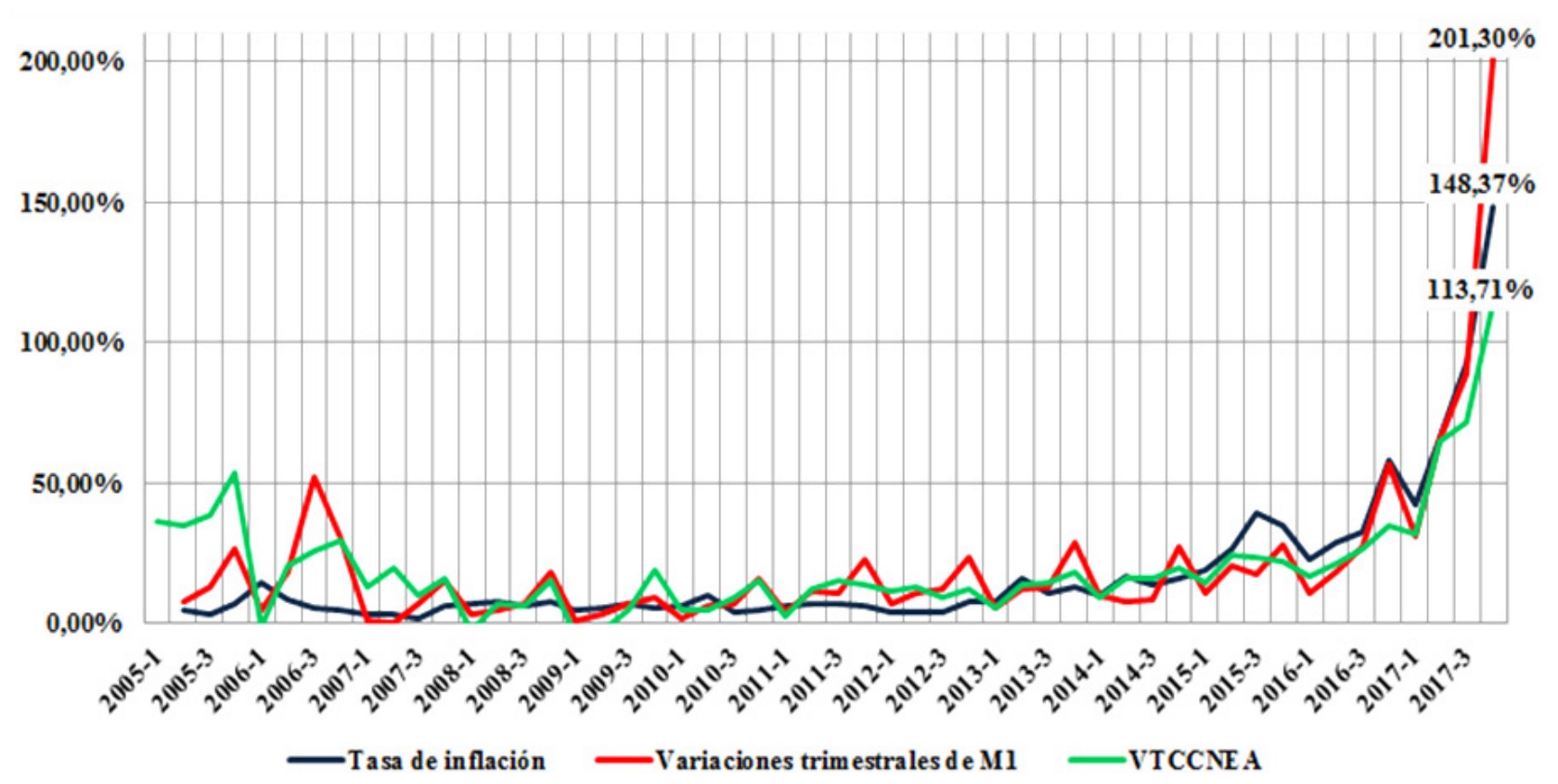

Figura 2. Variaciones trimestrales del INPC, CCNEA y M1 (período 2005-2017). Fuente: Estimaciones propias a partir de SUDEBAN (2017) y BCV (2017).

Lo antes expuesto ha sido producto de una política monetaria de carácter expansivo que ha instrumentado el Banco Central de Venezuela (BCV, 2018), que ha impulsado el incremento general de los precios y la expansión artificial del crédito a través del sistema financiero (Urdaneta, 2017).

Esta situación puede observarse en la Figura 4 donde el multiplicador monetario según Friedman y Meiselman (1963), es el mecanismo por el cual se produce la creación de dinero mediante reserva fraccionaría de depósitos bancarios, generando un crecimiento de la masa monetaria en circulación y mostrando una pendiente negativa denotando con ello que la velocidad a la que crece la Base Monetaria (BM) es superior al de la liquidez monetaria (M2), implicando un aumento del coeficiente de caja estimado por ser este el inverso del multiplicador monetario según Friedman y Meiselman (1963). 
En consecuencia, lo anterior representaría un menor margen de intermediación financiera y una restricción mayor al crédito, principio que no es aplicado por las políticas del Banco Central de Venezuela. Ahora bien, en el caso de la velocidad de circulación del dinero (numéricamente se calcula como la relación entre el PIB nominal y el circulante M1) (Blanchard, 2005).

En la Figura 3 puede evidenciarse como esta muestra una pendiente negativa como el multiplicador monetario, lo cual da indicios de que la velocidad a la que crece la liquidez monetaria (M2) es superior a la velocidad con que crece el volumen de las transacciones en la economía. Esta situación, denota indicios de poca dinámica económica (Friedman, 2005), donde el crecimiento de la producción de bienes y servicios a precios corrientes es inferior, al aumento de la masa monetaria.

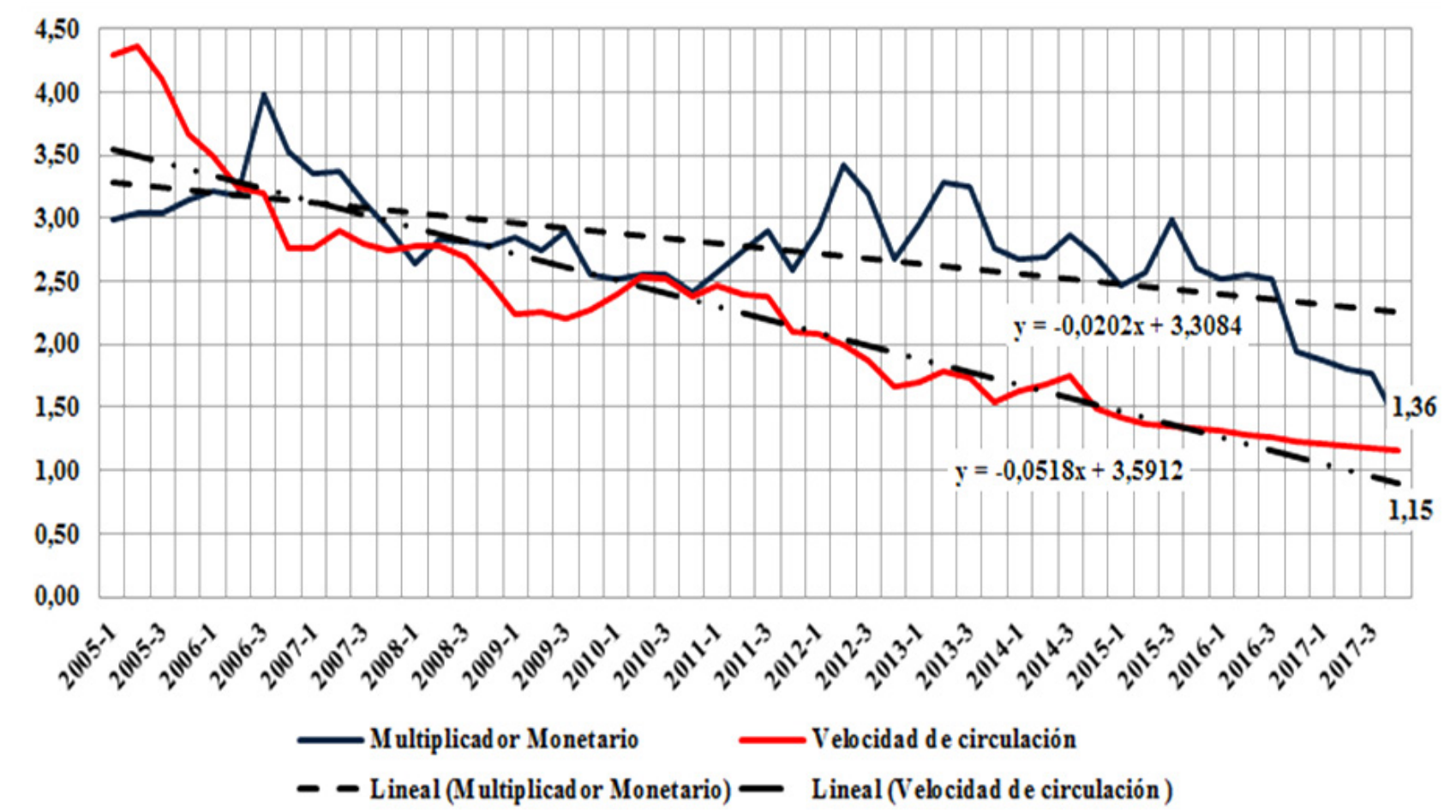

Figura 3. Multiplicador monetario y velocidad de circulación del dinero período 2005-2017 Fuente: Estimaciones propias a partir de BCV (2017).

En la Figura 4 se puede observar la evolución de agregados financieros medidos en términos reales, tomando como año base 2007. En el caso de los Depósitos a la Vista (DV), la Cartera de Créditos Neta Expandida Artificialmente (CCNEA) y la Disponibilidad Financiera (DF), las series de tiempo muestran un componente de tendencia ascendente y cíclico porque oscilan por encima y por debajo de la línea de tendencia en periodos superiores a un año.

En el caso de los DV estos presentan un ciclo expansivo que comienza en el trimestre 2011-4 y termina en el 2016-1. En dicho periodo, en términos reales, los mismos crecieron por encima de su valor promedio para el lapso 2005-2017. De igual forma la CCNEA muestra un crecimiento similar para el lustro 2011-3 al 2016-3, la misma se incrementó por arriba de su media para el lustro 2005-2017. 


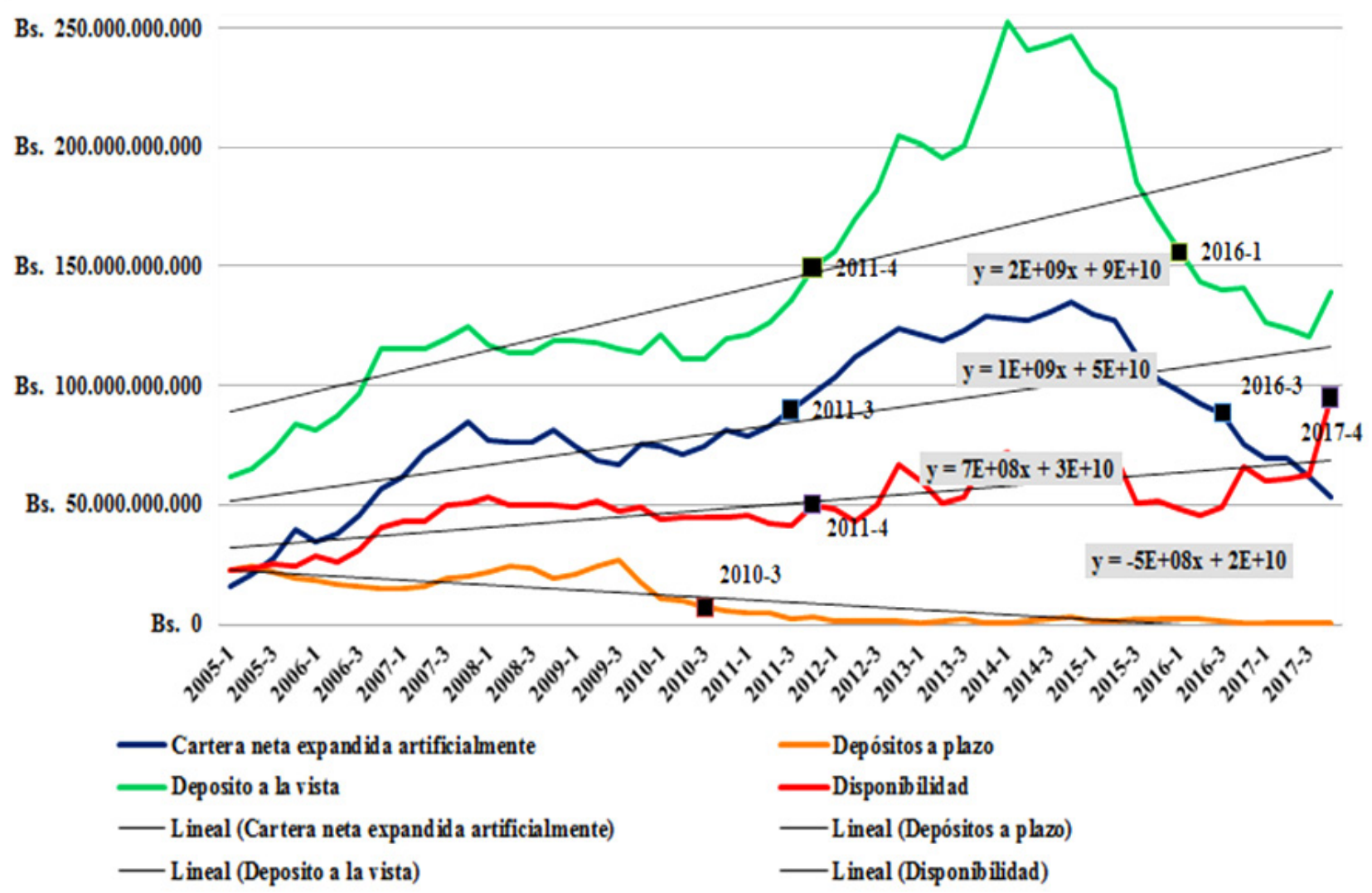

Figura 4. Agregados financieros a precios constantes año base 2007, período 2005-2017. Fuente: Estimaciones propias a partir de SUDEBAN (2017) y BCV (2018).

En ese mismo orden ideas, la DF, comenzó a crecer por encima de su valor promedio a partir del trimestre 2011-4 hasta el 2017-4, cuando supera a la CCNEA, producto de la contracción del crédito en términos reales, debido a la recesión económica existente (Urdaneta, Prieto y Hernández, 2017).

No obstante, mientras todos los agregados analizados en términos reales presentan un componente tendencial ascendente, los depósitos a plazo (DP), muestran componente tendencial descendente mostrando una pendiente negativa y cíclica porque oscilan por encima y por debajo de la línea de tendencia en periodos superiores a un año.

Sin embargo, los mismos DP a partir del trimestre 2010-3 comienzan una abrupta caída por debajo de su media para el periodo 2005-2017, llegando a ser pocos representativos para el análisis; más allá de que su desplome evidencie una mayor expansión artificial del crédito, el cual, al cierre de 2017-4, se financiaba con el 99,69\% de depósitos a la vista, mientras que en 2005-1 con el 41,37\%. Como se observa en el Figura 6, a partir del trimestre 2010-3 el porcentaje de la Cartera de Créditos Neta (CCN) que se financiada con DV era superior a su media para el lustro 2005-2017.

Todo lo anterior en un contexto en donde el índice de intermediación financiera (CCN/CP) se mantuvo oscilante entre el 45\% y 62\% desde 2005-1 hasta 2016-3, comenzando en 2016-4 con una caída abrupta hasta 2017-4 donde cerró en 36,20\%, producto de la contracción del crédito antes señala anteriormente. 


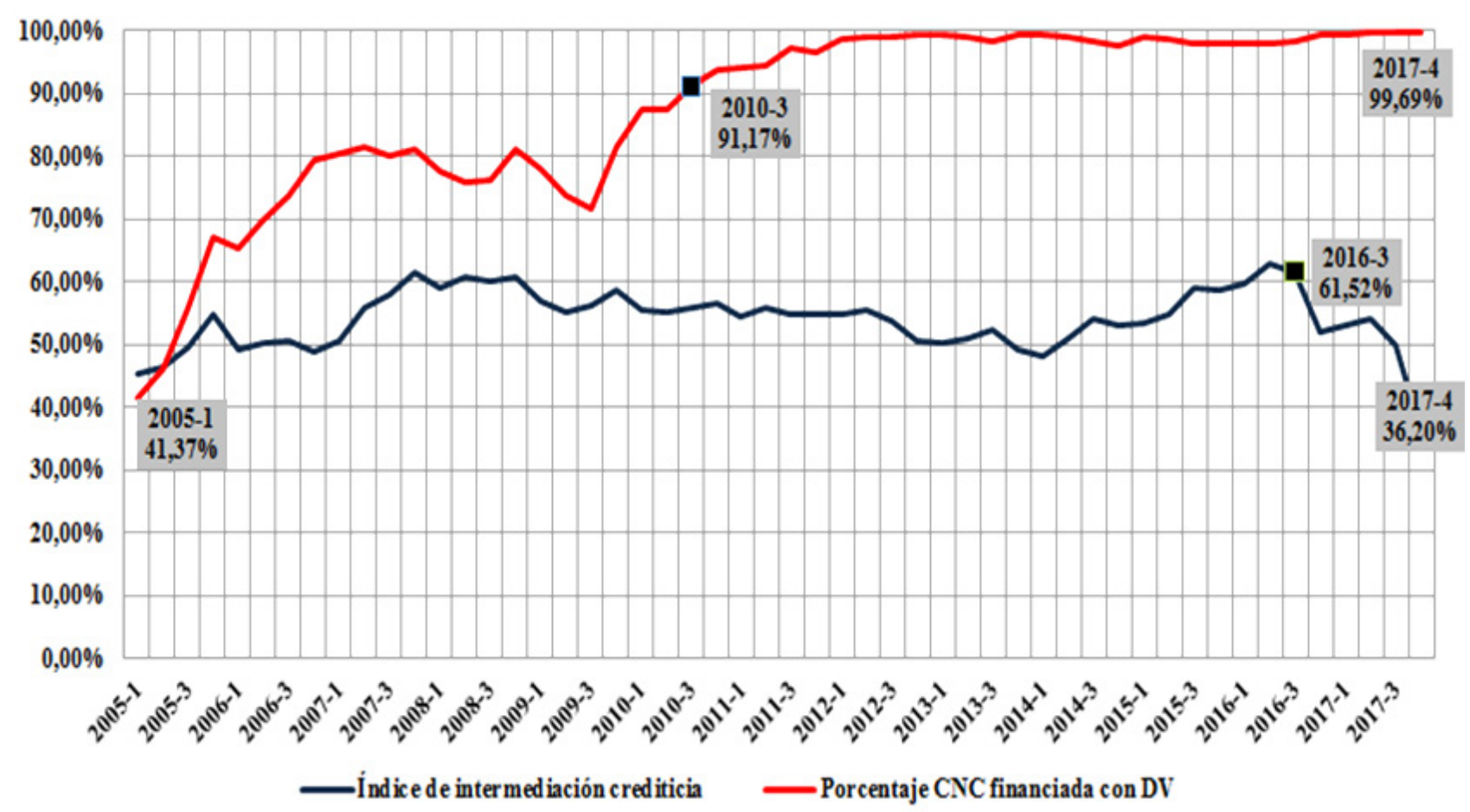

Figura 5. Índices financieros período 2005-2017

Fuente: Estimaciones propias a partir de SUDEBAN (2017).

Lo mostrado en la Figura 5 es producto de la expansión artificial del crédito, incluso al no ajustarse el BCV al coeficiente de caja estimado (CCE) bajo el sistema de reserva fraccionaria, como se muestra en la Figura 6, genera una mayor expansión artificial crediticia, donde el (CCE) se encuentra muy encima del establecido por el BCV como encaje legal para el sector bancario bajo el sistema de reserva fraccionaria.

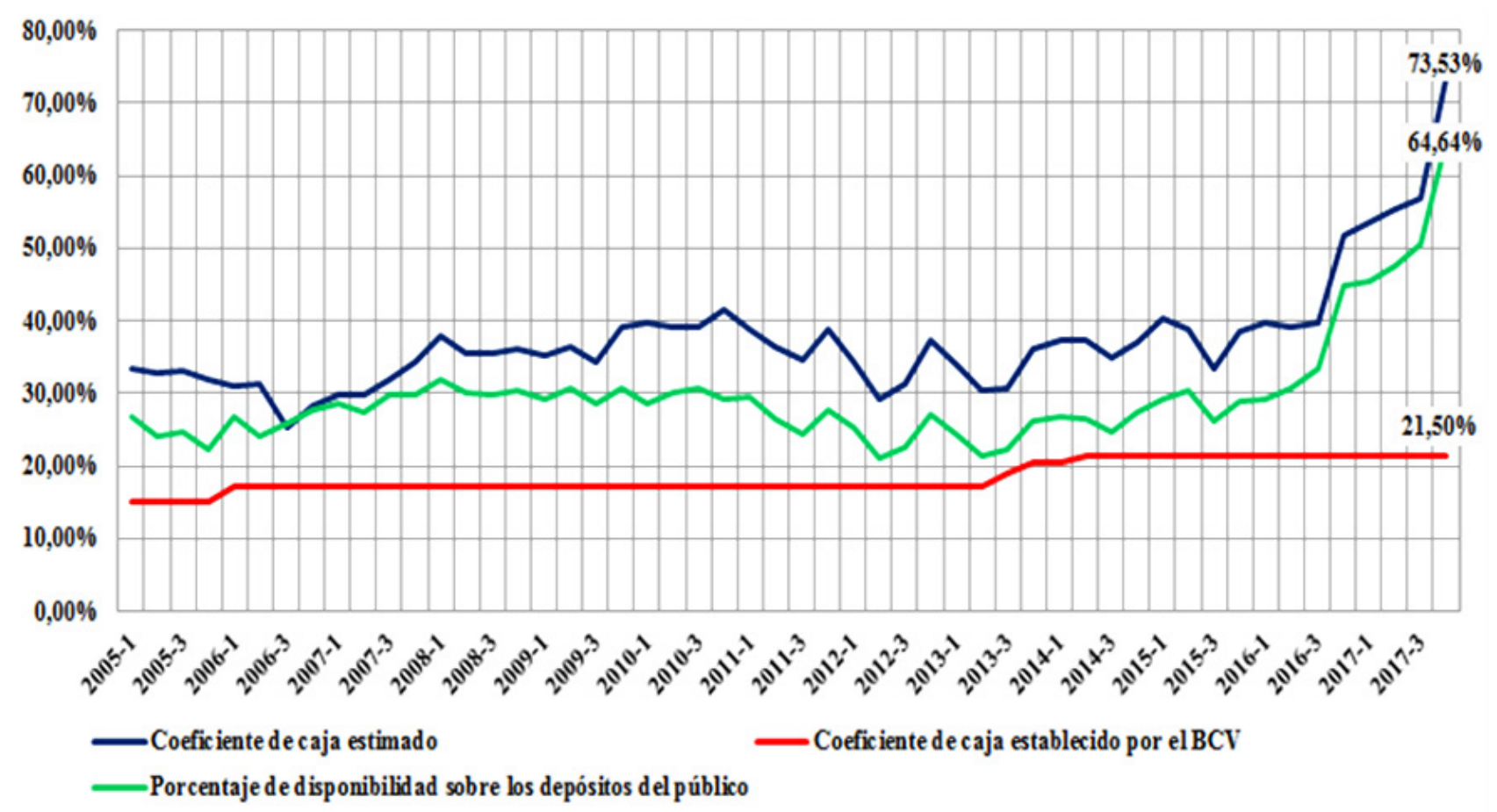

Figura 6. Índices financieros período 2005-2017

Fuente: Estimaciones propias a partir de SUDEBAN (2017) y BCV (2017). 
Sin embargo, debido al incremento exponencial de las Captaciones del Público (CP) y los DV, más no así de los DP, pasaron a ser en términos reales superiores a M2 (Figura 7). En el caso de las CP a partir del trimestre 2008-1, a inicios del proceso de reconversión monetaria; mientras en los DV a partir del trimestre 2014-1. Todo lo anterior se tradujo en que la DF comenzó a crecer en similares proporciones al CCE, debido a la contracción progresiva del crédito y la actividad económica, así como también a la imposición de gavetas crediticias obligatorias por parte de la SUDEBAN al sector, las cuales presentan una mayor contracción y una superior tasa de interés real negativa (Figura 7).

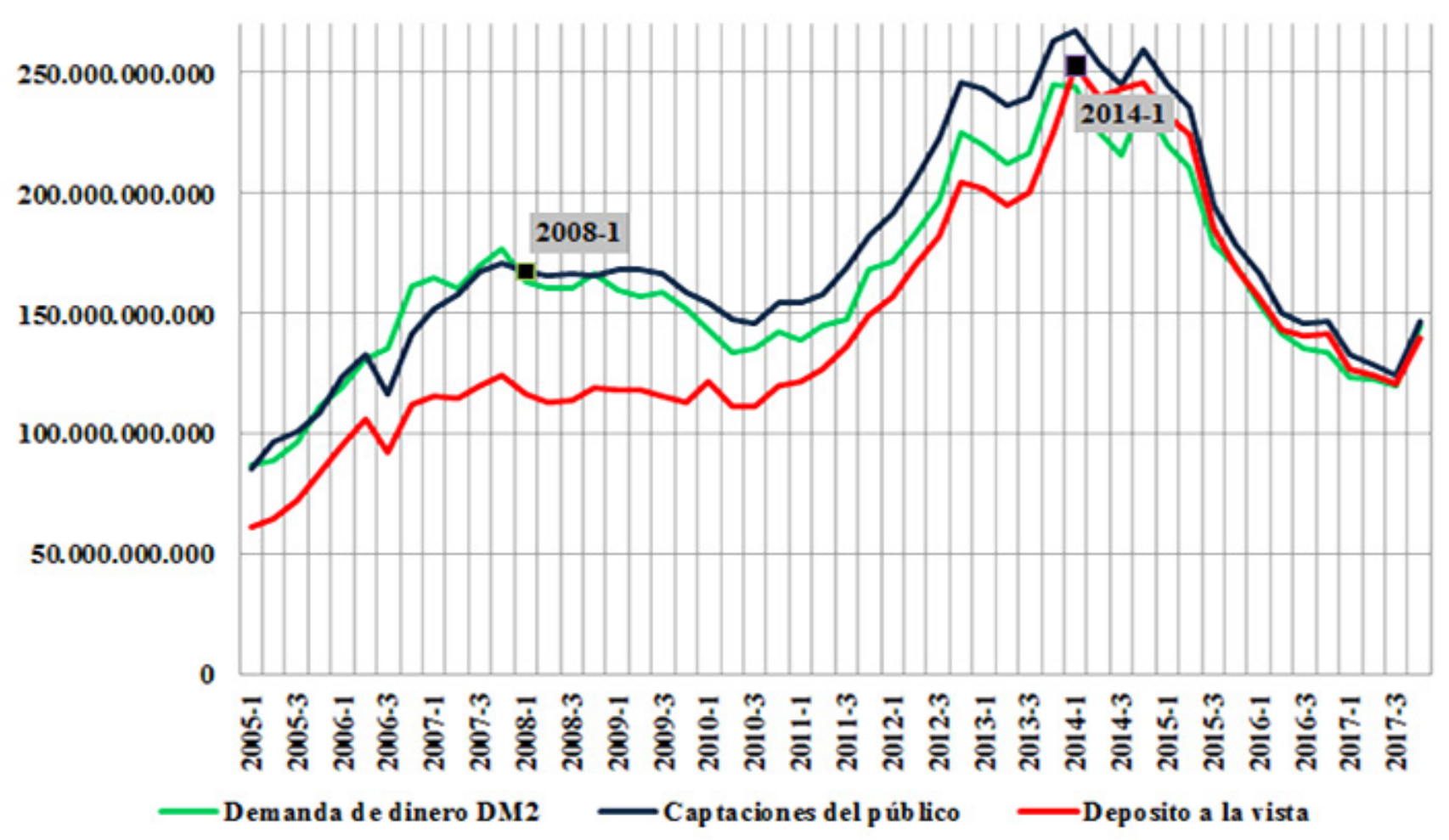

Figura 7. Agregados monetarios a precios constantes año base 2007, período 2005-2017 Fuente: Estimaciones propias a partir de SUDEBAN (2017) y BCV (2017).

En la Figura 7 se puede observar como en el lapso 2005-1 al 2007-4 los agregados monetarios medidos a precios constantes muestran una evolución acorde con lo que dicta la lógica de una política monetaria coherente, racional y creíble; es decir $(\mathrm{M} 2 / \mathrm{P}) \geq(\mathrm{CP} / \mathrm{P})$, pero a partir de 2008-1 con el inicio de la reconversión monetaria se revirtió el proceso $(\mathrm{M} 2 / \mathrm{P})<(\mathrm{CP} / \mathrm{P})$ aunque la lógica económica plantea que $(\mathrm{DV} / \mathrm{P})<(\mathrm{M} 2 / \mathrm{P})$. El esquema anterior se cumplió en la economía venezolana en el período 2005-1 al 2013-4; no obstante, a partir del trimestre 2014-1 los $(\mathrm{DV} / \mathrm{P})>(\mathrm{M} 2 / \mathrm{P})$ hasta el cierre de 2017-4.

La situación anterior permitió comprobar la tesis planteada por la teoría austriaca del dinero y el crédito de Mises (1982) al realizar el estudio con series de tiempo de las Variaciones Trimestrales de la Base Monetaria (VTBM), Liquidez Monetaria (VTM1), Cartera Neta de Crédito (VTCCN), el Índice Nacional de Precios al Consumidor (VTINPC) y la Cartera de Crédito Expandida Artificialmente (VTCCNEA), como variables endógenas, empleando Vectores AutoRegresivos Estructurados (VARE). 
En este estudio se emplearon modelos de vectores SVAR, los cuales para Urdaneta et al. (2017) y Chiang y Wainwright (2006) son vectores autorregresivos que representan modelos que se utilizan al caracterizar las interacciones simultáneas entre un grupo de variables. El VAR representa un modelo de ecuaciones simultáneas de forma reducida sin restringir, de modo que los valores contemporáneos de las variables del modelo no aparecen como variables explicativas en ninguna de las ecuaciones. Por el contrario, el conjunto de variables explicativas de cada ecuación está constituido por un bloque de retardos de cada una de las variables del modelo. Que sean ecuaciones no restringidas significa que aparece en cada una de ellas el mismo grupo de variables explicativas. En tal sentido, partiendo de un modelo estructural dinámico (1):

$$
y_{t}=\alpha y_{t-1}+\beta x_{t-1}+c
$$

Donde, $a$ y $\beta$ son los coeficientes de las variables endógenas de la ecuación, mientras $Y_{\mathrm{t}}$ es la variable endógena en el período actual. Por otra parte, $Y_{\mathrm{t}-1}$ es la misma variable endógena, pero con 1 período de rezago, $X_{\mathrm{t}-1}$ es la variable exógena con 1 período de rezago y $C$ es el intercepto. El modelo (1) se utilizó en la presente investigación para estimar el comportamiento VTINPC y VTCCNEA en función del saldo de las VTBM, VTCCN y VTM1 con la finalidad de encontrar las ecuaciones que más se ajusten al comportamiento de las variables endógenas.

TABLA 2.

Prueba de Raiz Unitaria Dickey-Fuller Aumentado

\begin{tabular}{|c|c|c|c|c|c|c|c|c|}
\hline \multirow{2}{*}{$\begin{array}{l}\text { Augmented Dickey. } \\
\text { Fuller test statistic }\end{array}$} & \multicolumn{4}{|c|}{ "MacKinnon (1996) one-sided p-values. } & \multirow{2}{*}{\multicolumn{4}{|c|}{ Statistics }} \\
\hline & \multicolumn{4}{|c|}{ Test critical values: } & & & & \\
\hline $\begin{array}{c}\text { Variables } \\
\text { endógenas del } \\
\text { modelo SVAR }\end{array}$ & $1 \%$ level & $5 \%$ level & $10 \%$ level & Prob. & \multirow[t]{2}{*}{ R-squared } & \multirow{2}{*}{$\begin{array}{l}\text { Adjusted } \\
\text { R- } \\
\text { squared }\end{array}$} & \multirow[t]{2}{*}{ F-statistic } & \multirow[t]{2}{*}{$\begin{array}{l}\text { Durbin- } \\
\text { Watson stat }\end{array}$} \\
\hline t-Statistic & $-3,5744$ & $-2,9238$ & $-2,5999$ & 0,05 & & & & \\
\hline VTBM & & 9,2159 & & 0,0000 & 0,7905 & 0,7710 & 40,5701 & 1,6394 \\
\hline VTCCN & & 4,7283 & & 0,0000 & 0,3727 & 0,3460 & 13,9617 & 1,8703 \\
\hline VTCCNEA & & 3,4321 & & 0,0014 & 0,5594 & 0,5057 & 10,4112 & 1,9329 \\
\hline VTINPC & & 4,3504 & & 0,0000 & 0,3757 & 0,2958 & 5,9348 & 1,8457 \\
\hline VTMl & & 3,8082 & & 0,0042 & 0,4969 & 0,4925 & 4,5859 & 1,7883 \\
\hline
\end{tabular}

Fuente: Elaboración propia.

En la Tabla 2 puede observarse como los estadísticos de prueba estimados en Eviews 6.0 para las variables sujetas estudios para los modelos (VARE) en valores absolutos son superiores que valores los críticos de McKinnon, al 1\%, 5\% y 10\%, razón por la cual todas las variables endógenas del modelo no presentan raíz unitaria y son estacionales en primera diferencia. Igualmente, si las probabilidades (Prob.) $<0,05$ como en este caso para las variables endógenas del modelo, se rechaza Ho por lo cual las mismas sirven para explicar el modelo (VARE). 
$\mathrm{Al}$ observar la bondad del ajuste mediante el $\mathrm{R} 2$, las variables tienen los siguientes valores: VTBM $0,7905 \approx 79 \%$, VTCCN $0,3727 \approx 37 \%$, VTCCN $0,5594 \approx 56 \%$, VTINPC $0,3757 \approx 38 \%$ y VTM1 0,4969 $\approx 50 \%$. El R2 ajustado: Permite medir el incremento neto de $\mathrm{R} 2$ cuando se incluye un nuevo regresor $Y(\mathrm{t}-2)$. En este caso, los valores fueron los siguientes: las VTBM0, $7710 \approx 77 \%$; VTCCN 0,3460 $\approx 34 \%$, VTCCN $0,5057 \approx 51 \%$, VTINPC 0,2958 $30 \%$ y VTM1 0,4925 $249 \%$.

Con relación a la prueba de hipótesis conjunta de los parámetros asociados, Fstatistic, son iguales a cero (excepto el intercepto). Siendo $H 0: \beta 1=\beta 2=\beta 3=\beta \mathrm{i}$, para un grado de libertad $g l=51-1=50$ y un nivel de confianza del $95 \%$, el valor calculado es F0, $05=4,08$ el cual es menor que los valores de F de prueba. De esta manera: 40,57 para VTBM; 13,96 para VTCCN; 10,41 para VTCCNEA; 5,93 para VTINPC; y 4,59 para VTM1. Estos resultados confirman la hipótesis planteada de no presencia de raíz unitaria.

Con respecto al estadístico Durbin-Watson (DW), el cual sirve para contrastar la hipótesis de la no-correlación serial entre perturbaciones aleatorias, mostró que los valores calculados para las variables consideradas fueron de: 1,64 para VTBM; 1,87 para VTCCN; 1,93 para VTCCNEA; 1,85 para VTINPC; y 1,79 para VTM1. Confirmando la hipótesis de incorrelación entre las variables endógenas.

\section{Prueba de causalidad}

La prueba de causalidad Granger es un test que buscar estimar cómo los resultados de una variable pueden predecir a otra, y si dicha relación causal es unidireccional o bidireccional. Ahora bien, en la Tabla 3 se muestra los resultados correspondientes con un nivel significación de $95 \%$ realizados a los vectores autorregresivos de las variables endógenas que se desean pronosticar VTINPC y VTCCNEA con un rezago ideal de 4 trimestres.

TABLA 3.

Prueba de Causalidad de Granger para las Variables Endógenas

\begin{tabular}{|c|c|c|c|c|c|c|c|}
\hline \multicolumn{8}{|c|}{ VAR Granger Causality/Block Exogeneity Wald Tests } \\
\hline \multicolumn{8}{|c|}{ Date: 01/28/18 Time: 10:03 } \\
\hline \multicolumn{8}{|c|}{ Sample: 2005Q1 2017Q4 } \\
\hline \multicolumn{8}{|c|}{ Included observations: 48} \\
\hline \multicolumn{4}{|c|}{ Dependent variable: VTCCNEA } & \multicolumn{4}{|c|}{ Dependent variable: VTINPC } \\
\hline Excluded & Chi-sq & df & Prob. & Excluded & Chi-sq & df & Prob. \\
\hline VTBM & 22,9458 & 4 & 0,0001 & VTCCNEA & 7,0287 & 4 & 0,1344 \\
\hline VTCCN & 12,8785 & 4 & 0,0119 & VTBM & 23,4225 & 4 & 0,0001 \\
\hline VTINPC & 6,3423 & 4 & 0,1750 & VTCCN & 3,0694 & 4 & 0,5463 \\
\hline VTMl & 4,4865 & 4 & 0,3442 & VTMl & 10,4011 & 4 & 0,0342 \\
\hline All & 17,8023 & 16 & 0,0000 & All & 46,1626 & 16 & 0,0001 \\
\hline
\end{tabular}

Fuente: Elaboración propia. 
Según la prueba de LagOrderSelectionCriteria, en la cual el estadístico $x 2$ (ChiCuadrado teórico) $=9,49$ para cuatro grados de libertad, demuestran, en el caso de las VTINPC, VTBM y VTM1 la existencia de una relación causal debido a que el $x^{2}$ (Chi-Cuadrado calculado) VTBM $=23,4225>9,49$ y $x 2$ (Chi-Cuadrado calculado) VTM1 = 10,4011 > 9,49 (Tabla 2) y sus Prob. < 0,05; mas no así con VTCCN y VTCCNEA.

Igualmente se evidenció, en el caso de las VTCCNEA, VTBM y VTCCN que existe una relación causal debido al $\mathrm{X}^{2}$ (Chi-Cuadrado calculado) $\mathrm{VTBM}=22,9458>9,49 \mathrm{y}$ $x 2$ (Chi-Cuadrado calculado) VTCCN $=12,8785>9,49$ (Tabla 2) y sus Prob. $<0,05$; mas no así con VTINPC y VTM1. En consecuencia, los valores rezagados de VTBM y VTM1 ayudan hacer un mejor pronóstico de VTINPC y los valores rezagados de, VTBM y VTCCN permiten realizan un mejor pronóstico de VTCCNEA.

TABLA 4.

Prueba de exclusión de rezagos no significativos

\begin{tabular}{|c|c|c|c|c|}
\hline \multicolumn{5}{|c|}{ VAR Lag Exclusion Wald Tests } \\
\hline \multicolumn{5}{|c|}{ Date: $01 / 19 / 18$ Time: $11: 54$} \\
\hline \multicolumn{5}{|c|}{ Sample: 2005Q1 2017Q4 } \\
\hline \multicolumn{5}{|c|}{ Included observations: 51} \\
\hline \multicolumn{5}{|c|}{ Chi-squared test statistics for lag exclusion: } \\
\hline \multicolumn{5}{|c|}{ Numbers in [ ] are p-values } \\
\hline & VTINPC & VTBM & VTM1 & Joint \\
\hline \multirow[t]{2}{*}{ Lag 1} & 217,668400 & 89,283420 & 103,059000 & 287,583200 \\
\hline & {$[0.000000]$} & {$[0.000000]$} & {$[0.000000]$} & {$[0.000000]$} \\
\hline df & 3 & 3 & 3 & 9 \\
\hline \multicolumn{5}{|c|}{ VAR Lag Exclusion Wald Tests } \\
\hline \multicolumn{5}{|c|}{ Date: $01 / 19 / 18 \quad$ Time: $11: 57$} \\
\hline \multicolumn{5}{|c|}{ Sample: 2005Q1 2017Q4 } \\
\hline \multicolumn{5}{|c|}{ Included observations: 50} \\
\hline \multicolumn{5}{|c|}{ Chi-squared test statistics for lag exclusion: } \\
\hline \multicolumn{5}{|c|}{ Numbers in [ ] are p-values } \\
\hline & VTCCNEA & VTBM & VTCCN & Joint \\
\hline \multirow[t]{2}{*}{ Lag 1} & 15,750800 & 9,678830 & 31,856640 & 55,392020 \\
\hline & {$[0.001275]$} & {$[0.021503]$} & {$[5.61 \mathrm{e}-07]$} & {$[1.03 \mathrm{e}-08]$} \\
\hline \multirow[t]{2}{*}{ Lag 2} & 21,458150 & 15,681870 & 36,405850 & 63,481350 \\
\hline & [8.46e-05] & {$[0.001318]$} & [6.15e-08] & {$[2.85 \mathrm{e}-10]$} \\
\hline $\mathrm{df}$ & 3 & 3 & 3 & 9 \\
\hline
\end{tabular}

Fuente: Elaboración propia.

En la Tabla 4 se muestra como los $p$-value (paréntesis) son menores al 5\% para todas las variables endógenas de los modelos SVAR en el caso del pronóstico de la variable endógena VTINPC se tomó un solo rezago, porque todo los p-value son iguales a [0,000000], lo cual es altamente significativo. En el caso VTCCNEA se tomaron dos rezagos, ya que en la prueba de cointegración de Johansen el criterio de información Akaike indica que a partir del segundo rezago el modelo es significativo. Con base a lo anterior se estimaron los siguientes (VARE), mostrados en la Tabla 5 . 
TABLA 5.

Modelo de Vectores Autorregresivos Estructurados

\begin{tabular}{|c|c|c|c|}
\hline \multicolumn{2}{|c|}{ Vector Autoregression Estimates } & \multirow{2}{*}{$\begin{array}{c}\text { VTCCNEA } \\
0,7635\end{array}$} & \multirow{2}{*}{$\begin{array}{c}\text { VTINPC } \\
0,8224\end{array}$} \\
\hline & R-squared & & \\
\hline $\begin{array}{c}\text { Standard errors in () } \\
\& \text { t-statistics in [] }\end{array}$ & $\begin{array}{c}\text { Adj. R- } \\
\text { squared }\end{array}$ & 0,7304 & 0,8111 \\
\hline \multicolumn{4}{|c|}{ Modelos de Vectores Autorregresivos } \\
\hline \multicolumn{4}{|c|}{$\begin{array}{c}V T I N P C=1,36891335045^{\star} V T I N P C(-1)+ \\
0,0628877738429^{*} V T B M(-1)-0,161073151108^{*} V T M 1(-1)- \\
0,0104923200681\end{array}$} \\
\hline \multicolumn{4}{|c|}{$\begin{array}{c}V T C C N E A=0,218892128956^{*} V T C C N E A(-1)- \\
0,281145331886^{*} V T C C N E A(-2)-0,152301390797^{*} V T B M(-1)+ \\
0,107596222425^{*} V T B M(-2)+0,53812200006^{*} V T C C N(-1)+ \\
1,16816733514^{*} V T C C N(-2)-0,0413497897283\end{array}$} \\
\hline
\end{tabular}

Fuente: Elaboración propia.

En la Tabla 5 se muestran los valores de R2 los cuales expresan el porcentaje de la varianza de las variables endógenas VTINPC y VTCCNEA, que es explicado por los modelos de vectores autorregresivos, $82,24 \%$ y $76,35 \%$ respectivamente, mientras que él R2-ajustado explica el incremento de la proporción de la varianza que es explicado por el modelo (VARE) al ingresar un nuevo rezago.

\section{Análisis de Cointegración de variables}

Se dice que dos o más series están cointegradas si las mismas se mueven conjuntamente a lo largo del tiempo y las diferencias entre ellas son estables (es decir estacionarias), aun cuando cada serie en particular contenga una tendencia estocástica y sea por lo tanto no estacionaria. De aquí que la cointegración refleja la presencia de un equilibrio a largo plazo hacia el cual converge el sistema económico. Las diferencias (o término error) en la ecuación de cointegración se interpretan como el error de desequilibrio para cada punto particular de tiempo.

En la Tabla 6 se observan 13 vectores de cointegración para la prueba de las trazas y 10 para la prueba de máximo valor propio para el pronóstico de la variable endógena VTINPC. Por otra parte, para la variable endógena VTCCNEA, se evidencian 13 vectores de cointegración tanto para la prueba de las trazas, como para la prueba de máximo valores propios.

Para los efectos del presente estudio se generaron 5 opciones de relaciones de cointegración que genera el modelo tales como: sin intercepto y sin tendencia, con intercepto y sin tendencia, lineal con intercepto y sin tendencia, lineal con intercepto y tendencia y cuadrática con intercepto y tendencia. De todas las opciones, se tomaron para ambos (VARE) la relación de cointegración con vectores con intercepto y sin tendencia como se muestran a continuación en la Tabla 7 y Tabla 8, demostrando una relación a largo plazo entre las variables endógenas VTINPC, VTBM y VTM1; así como también VTCCNEA, VTBM y VTCCN. 
TABLA 6.

Resumen de la Prueba de Cointegración de Johansen

\begin{tabular}{|c|c|c|c|c|c|}
\hline \multicolumn{6}{|c|}{ Date: $01 / 28 / 18$ Time: $05: 50$} \\
\hline \multicolumn{6}{|c|}{ Sample: 2005Q1 2017Q4 } \\
\hline \multicolumn{6}{|c|}{ Included observations: 50} \\
\hline \multicolumn{6}{|c|}{ Series: VTINPC VTBM VTM1 } \\
\hline \multicolumn{6}{|c|}{ Lags interval: 1 to 1} \\
\hline \multicolumn{6}{|c|}{ Selected $\left(0.05\right.$ level $\left.{ }^{*}\right)$ Number of Cointegrating Relations by Model } \\
\hline Data Trend: & None & None & Linear & Linear & Quadratic \\
\hline \multirow[t]{2}{*}{ Test Type } & No Intercept & Intercept & Intercept & Intercept & Intercept \\
\hline & No Trend & No Trend & No Trend & Trend & Trend \\
\hline Trace & 3 & 2 & 3 & 2 & 3 \\
\hline Max-Eig & 3 & 2 & 3 & 1 & 1 \\
\hline \multicolumn{6}{|c|}{ Included observations: 49} \\
\hline \multicolumn{6}{|c|}{ Series: VTCCNEA VTBM VTCCN } \\
\hline \multicolumn{6}{|c|}{ Lags interval: 1 to 2} \\
\hline \multicolumn{6}{|c|}{ Selected $(0.05$ level*) Number of Cointegrating Relations by Model } \\
\hline Data Trend: & None & None & Linear & Linear & Quadratic \\
\hline \multirow[t]{2}{*}{ Test Type } & No Intercept & Intercept & Intercept & Intercept & Intercept \\
\hline & No Trend & No Trend & No Trend & Trend & Trend \\
\hline Trace & 3 & 2 & 3 & 2 & 3 \\
\hline Max-Eig & 3 & 2 & 3 & 2 & 3 \\
\hline & itical values be & on MacKir & aug-Miche & 99) & \\
\hline
\end{tabular}

Fuente: Elaboración propia.

TABLA 7.

Vector de cointegración para VTINPC, VTBM y VTM1

\begin{tabular}{|c|c|c|c|c|}
\hline \multicolumn{5}{|c|}{ Series: VTINPC VTBM VTM1 } \\
\hline \multicolumn{5}{|c|}{ Lags interval (in first differences): 1 to 1} \\
\hline \multicolumn{5}{|c|}{ Unrestricted Cointegration Rank Test (Trace) } \\
\hline Hypothesized & & Trace & 0.05 & \\
\hline No. of $\mathrm{CE}(\mathrm{s})$ & Eigenvalue & Statistic & Critical Vahue & Prob.** \\
\hline None * & 0,5460 & 68,2427 & 35,1928 & 0,0000 \\
\hline \multicolumn{5}{|c|}{ Trace test indicates 1 cointegrating eqn(s) at the 0.05 level } \\
\hline Hypothesized & & Max-Eigen & 0.05 & \\
\hline No. of $\mathrm{CE}(\mathrm{s})$ & Eigenvalue & Statistic & Critical Value & Prob.** \\
\hline None * & 0,5460 & 39,4814 & 22,2996 & 0,0001 \\
\hline \multicolumn{5}{|c|}{ Max-eigenvalue test indicates 1 cointegrating eqn $(\mathrm{s})$ at the 0.05 level } \\
\hline \multicolumn{5}{|l|}{1 Cointegrating } \\
\hline Equation(s): & & Log bikelihood & 117,1870 & \\
\hline \multicolumn{5}{|c|}{ Normalized cointegrating coefficients (standard error in parentheses) } \\
\hline VTINPC & VTBM & VTM1 & $\mathrm{C}$ & \\
\hline 1,000000 & 0,0660 & $-0,8700$ & $-0,0124$ & \\
\hline & $-0,107163$ & $-0,153012$ & $-0,17074$ & \\
\hline
\end{tabular}

Fuente: Elaboración propia. 
TABLA 8.

Vector de cointegración para VTCCNEA, VTBM y VTCCN

\begin{tabular}{|c|c|c|c|c|}
\hline \multicolumn{5}{|c|}{ Series: VTCCNEA VTBM VTCCN } \\
\hline \multicolumn{5}{|c|}{ Lags interval (in first differences): 1 to 1} \\
\hline \multicolumn{5}{|c|}{ Unrestricted Cointegration Rank Test (Trace) } \\
\hline Hypothesized & & Trace & 0.05 & \\
\hline No. of $\mathrm{CE}(\mathrm{s})$ & Eigenvalue & Statistic & Critical Value & Prob.** \\
\hline None * & 0,6710 & 85,6203 & 35,1928 & 0,0000 \\
\hline \multicolumn{5}{|c|}{ Trace test indicates 1 cointegrating eqn(s) at the 0.05 level } \\
\hline Hypothesized & & Max-Eigen & 0.05 & \\
\hline No. of $\mathrm{CE}(\mathrm{s})$ & Eigenvalue & Statistic & Critical Value & Prob.** \\
\hline None * & 0,6710 & 54,4657 & 22,2996 & 0,0000 \\
\hline \multicolumn{5}{|c|}{ Max-eigenvalue test indicates 1 cointegrating eqn(s) at the 0.05 level } \\
\hline \multicolumn{5}{|l|}{1 Cointegrating } \\
\hline \multicolumn{5}{|c|}{ Normalized cointegrating coefficients (standard error in parentheses) } \\
\hline VTCCNEA & VTBM & VTCCN & $\mathrm{C}$ & \\
\hline 1,000000 & 0,049134 & $-1,106942$ & $-0,016485$ & \\
\hline & $-0,096623$ & $-0,041773$ & $-0,013088$ & \\
\hline
\end{tabular}

Fuente: Elaboración propia.

En la Tabla 7 y Tabla 8 puede observarse como el estadístico de prueba Statistic > Critical value y Prob. ${ }^{* *}<0,05$ lo cual evidencia que los vectores de cointegración con sus coeficientes normalizados mostrados al final de cada tabla, permiten proyectar una relación a largo plazo entre dichas variables endógenas (Figura 8 y Figura 9) conjuntamente con las variables endógenas reales y proyectadas.

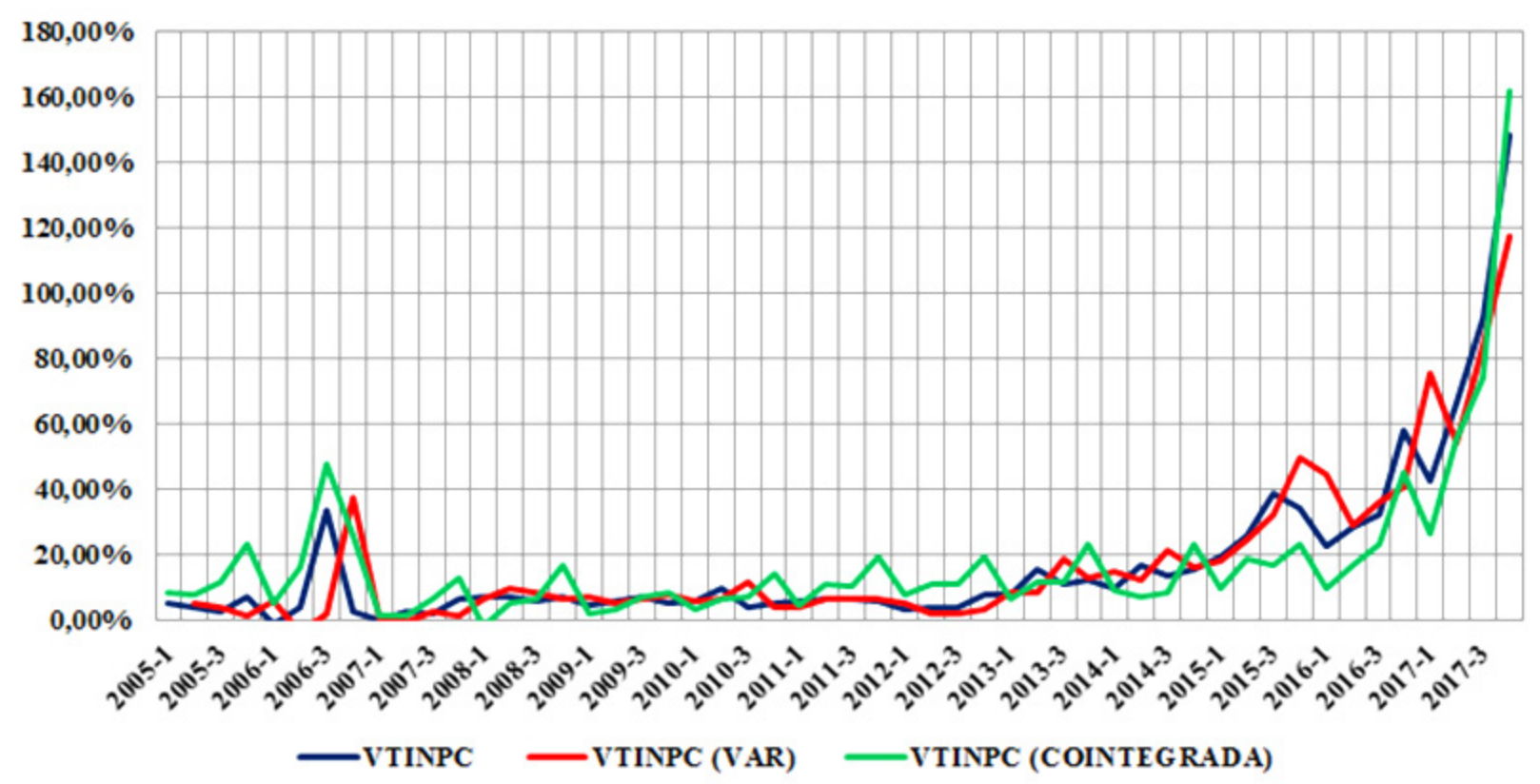

Figura 8. VTINPC, Vector autorregresivo para VTINPC y Vector de cointegración de VTINPC con VTBM y VTM1 para el período 2005-2017.

Fuente: BCV (2017). 


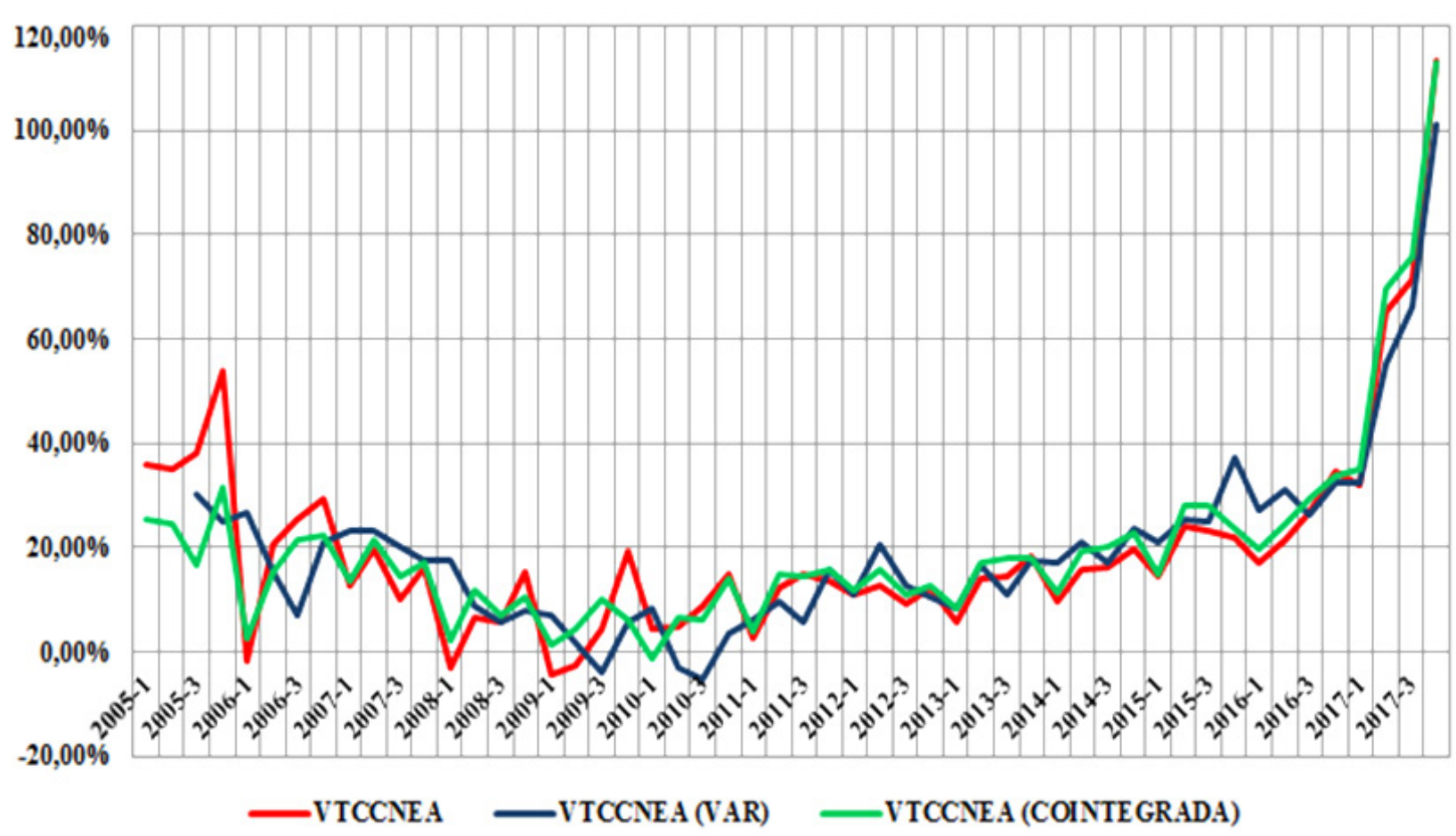

Figura 9. VTCCNEA, Vector autorregresivo para VTCCNEA y Vector de cointegración de VTCNEA con VTBM y VTCCN para el período 2005-2017 Fuente: BCV (2017).

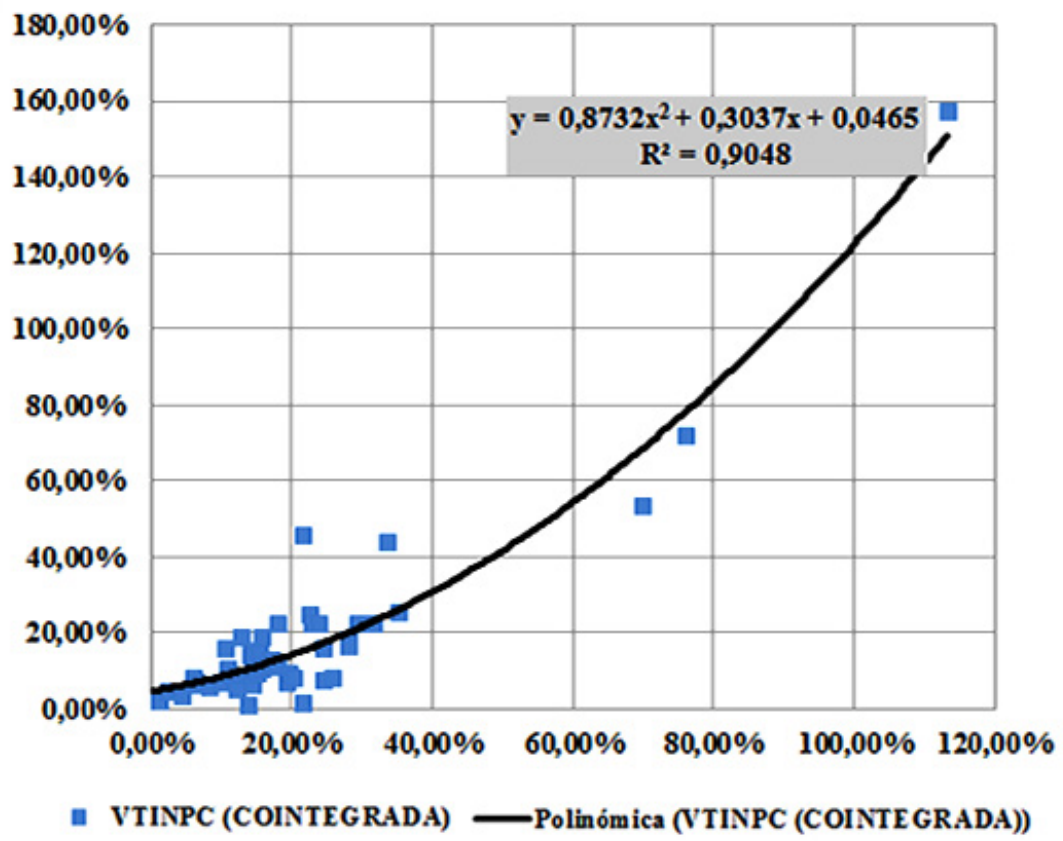

Figura 10. Vector de cointegración de VTINPC respecto al vector de cointegración de VTCCNEA para el período 2005-2017

Fuente: BCV (2017).

En la Figura 10 se observa la respuesta de variable endógena VTCCNEA al impulso causado por sus valores de rezago en el modelo de vector autorregresivo VTCCNEA $=0,218892128956{ }^{*}$ VTCCNEA $(-1)-0,281145331886{ }^{*}$ VTCCNEA (-2) $-0,152301390797{ }^{*} \operatorname{VTBM}(-1)+0,107596222425^{*} \operatorname{VTBM}(-2)+0,53812200006$ * VTCCN (-1) + 1,16816733514 * VTCCN (-2) - 0,0413497897283; a partir del período $t+1$; mientras que para las variables endógenas VTCCN y VTBM la respuesta se produce para el periodo $t+2$. 
La Figura 10 muestra la función resultante del vector de cointegración de VTINPC respecto al vector de cointegración de VTCCNEA para el período 20052017 , cuya línea de tendencia es $y=0,8732 \times 2+0,3037 x+0,0465$, el cual explica el 90,48\% de su varianza y un coeficiente de correlación lineal de Pearson del 0,95 indicando el alto grado de asociación lineal entre el vector de cointegración de VTINPC respecto al vector de cointegración de VTCCNEA, y un coeficiente de elasticidad de 1,49 con lo cual una variación de trimestral de 1\% de la cartera de créditos neta expandida artificialmente se traduce en un incremento del 1,49\% del índice nacional de precios al consumidor, demostrando en este caso un componente de elasticidad.

En ese mismo orden en la Figura 11 para la variable endógena VTINPC se evidencia una respuesta al impulso causado por sus valores de rezago en el modelo de vector autorregresivo VTINPC $=1,36891335045{ }^{*}$ VTINPC $(-1)+0,0628877738429$ *VTBM (-1) - 0,161073151108 *VTM1 (-1) - 0,0104923200681; a partir del período $t+1$; mientras que para las variables endógenas VTM1 y VTBM la respuesta es a partir del periodo $t+2$.

Response to Cholesky One S.D. Innovations \pm 2 S.E.

Response of VTCCNEA to VTCCNEA

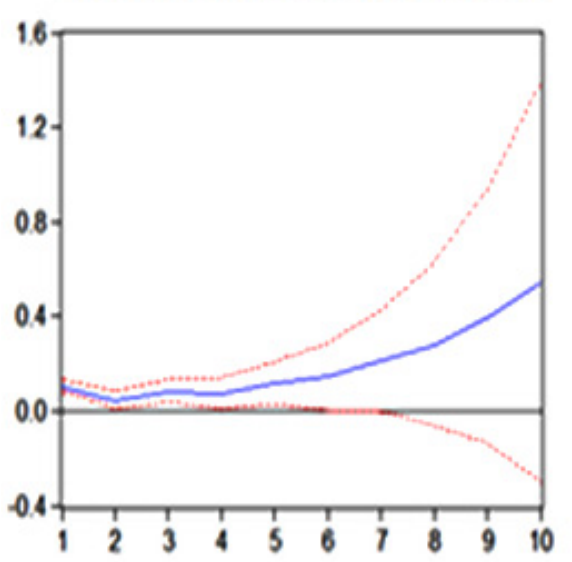

Response of VTCCNEA to VTBM

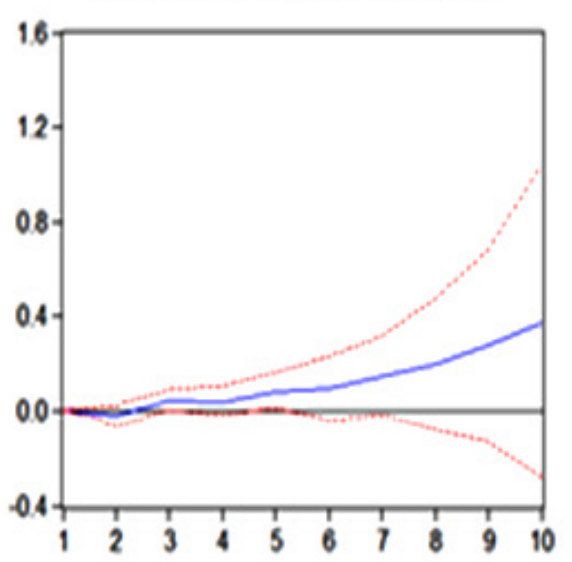

Response of VTCCNEA to VTCCN

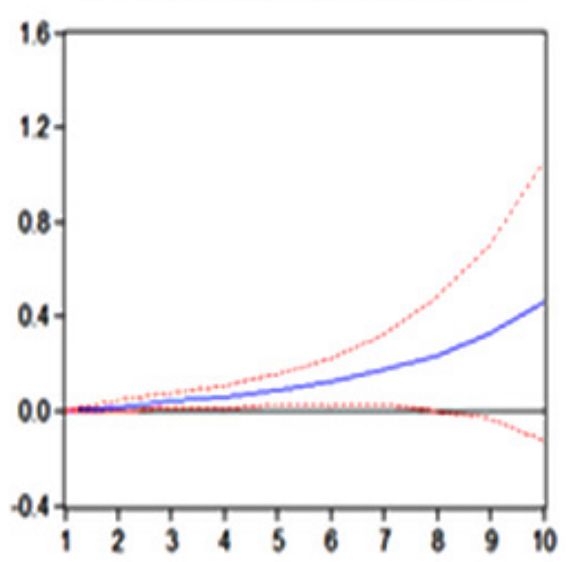

Response to Cholesky One S.D. Innovations \pm 2 S.E.

Response of VTINPC 10 VTINPC

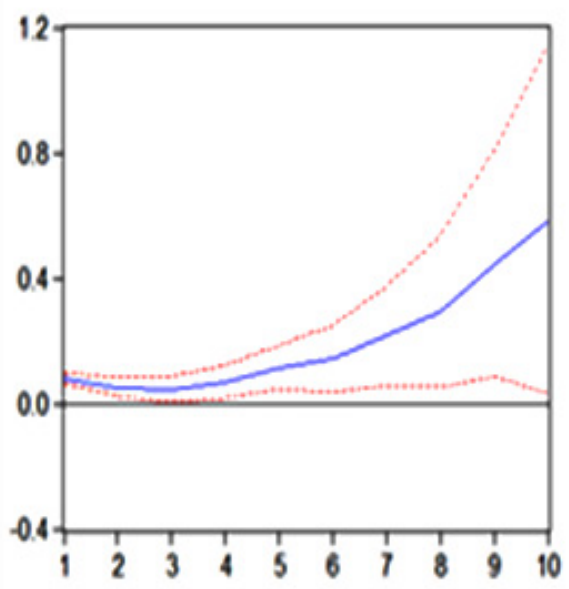

Response of VTINPC to VTBM

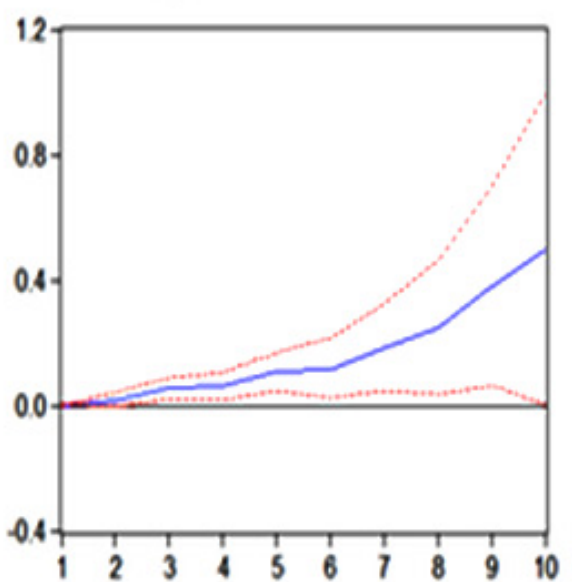

Response of VTINPC to VTM1

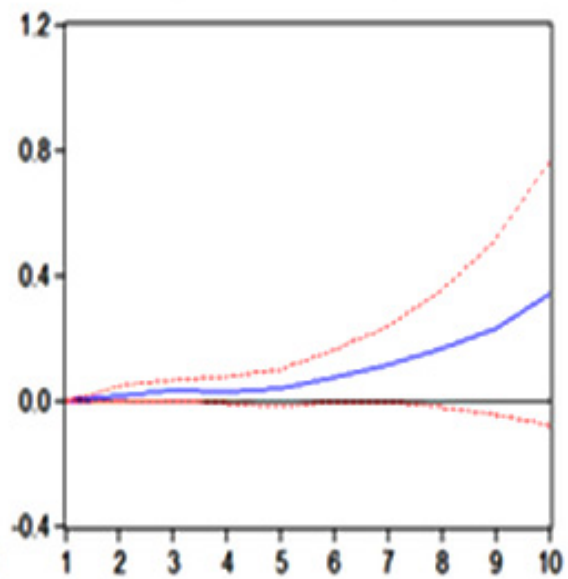

Figura 11. Función impulso respuesta para VTCCNEA y VTINPC para el período 2005-2017 Fuente: BCV (2017). 
En la Figura 12 se muestran los correlogramas para medir la posible autocorrelación de los residuos con error estándar a 2 bandas $\pm 0,288675$ tomando una muestra de 20 residuos, que representan $38,46 \%$ de los residuos totales, lo cual es significativo para la prueba que exige al menos el $30 \%$; se puede constatar como para las variables endógenas VTCCNEA y VTINPC con sus valores de rezago el 95\% o más están entre las bandas de tolerancia; mientras que para VTBM y VTCCN con respecto a VTCCNEA, así mismo de VTBM y VTM1 en relación a VTINPC, en ambos casos $100 \%$ de los residuos se encuentran dentro de las bandas de tolerancia, lo cual denota la no existencia de autocorrelación entre los residuos y se cumple la condición de independencia de los mismos.

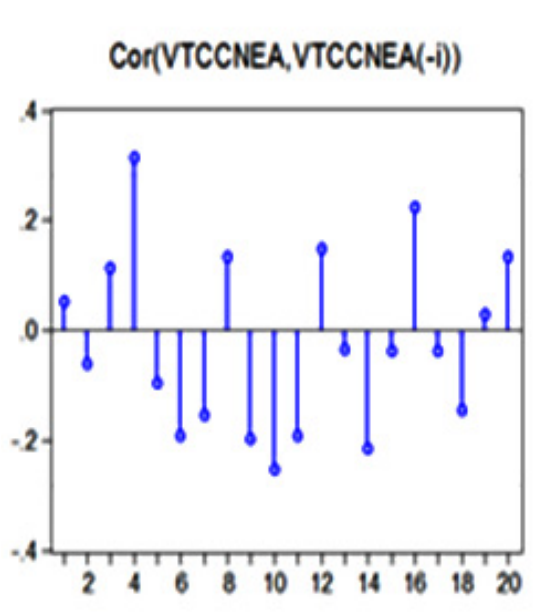

Autocorrelations with 2 Std.Err. Bounds
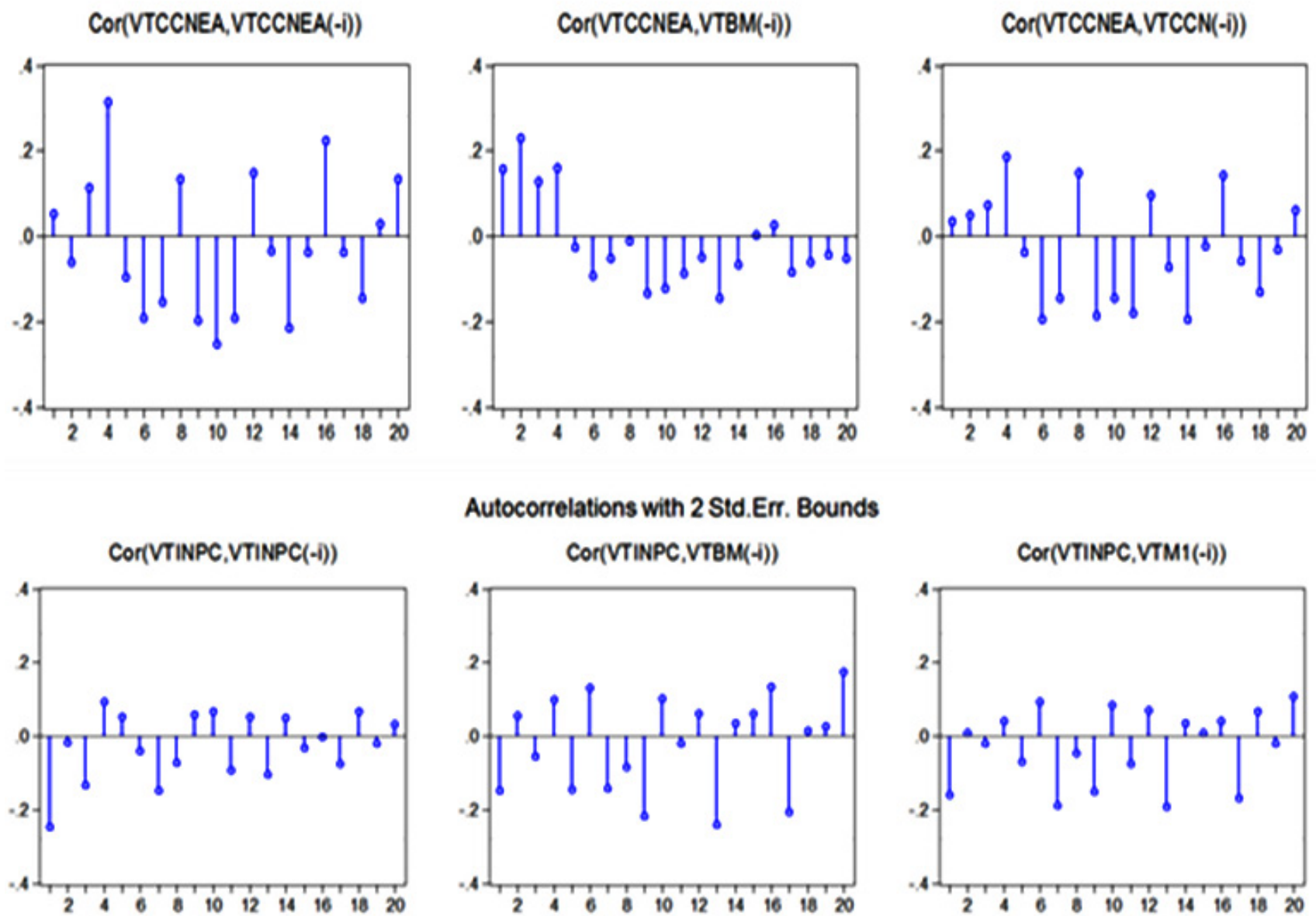

Autocorrelations with 2 Std.Err. Bounds
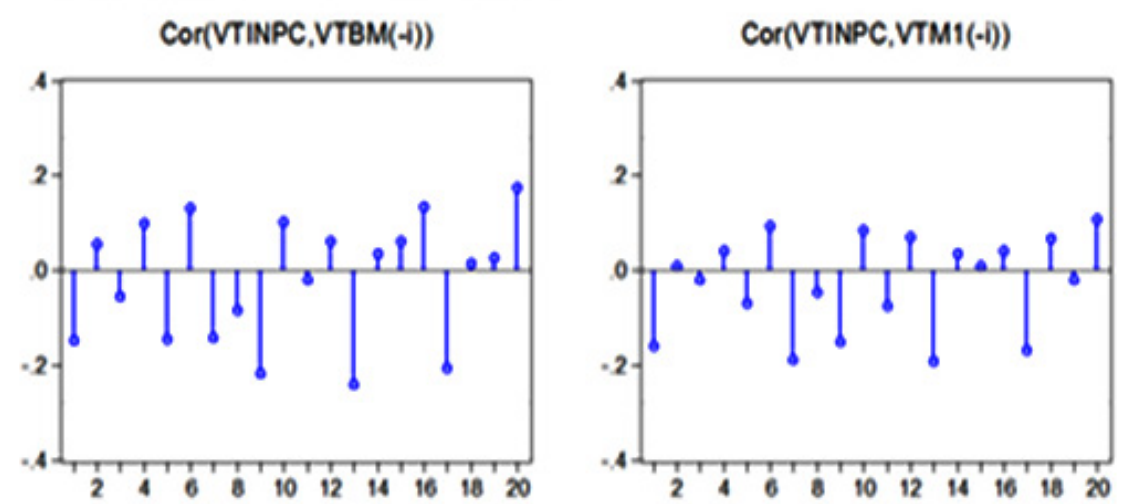

Figura 12. Correlogramas para VTCCNEA y VTINPC para el período 2005-2017 Fuente: BCV (2017).

\section{CONCLUSiOnes}

En el presente estudio puede sintetizarse como efectivamente la expansión artificial del crédito creció de manera exponencial en el período sujeto a estudio, pasando el financiamiento de la cartera de créditos neta con depósitos a la vista del $41,37 \%$ en el trimestre de 2005-1 al 99,69\% en 2017-4. Estos resultados se compaginan con la tasa de inflación anualizada al 2005-1, la cual era de 33,58\% (Baptista, 2011); mientras para el 2017-4 según la Asamblea Nacional la inflación anualizada fue de $2.616 \%$. 
Ciertamente, diversos estudios como el de Villar, Salamanca y Murcia (2005) han relacionado la expansión de crédito con el aumento de los flujos de capitales, como en el caso colombiano donde a través de una política de regulación financiera se ha creado un comportamiento prociclico de esta.

Lorente (2019) expresa como la expansión de los créditos tiene un importante impacto en el crecimiento e inflación, los cuales históricamente han sido hechos con gran variedad de traumas, consecuencia de la gestión de dichos recursos, creando crecimiento basado más en acumulación de riquezas que en la diversificación y mejora de los mercados o el desarrollo e innovación dentro de las industrias más competitivas.

La situación planteada en el caso venezolano se ha traducido, a lo largo del período estudiado, en una caída abrupta de los depósitos a plazo, los cuales para 2005-1 representaban el 26,95\% de los depósitos totales y para 2017-4 el 0,12\%. Esto se tradujo en un incremento superlativo de los depósitos a la vista pasando del 71,79\% de las captaciones del público en 2005-1 a 94,83\% en 2017-4. Tal realidad produjo que entre 2011-3 y 2016-3 se generara en términos reales, la conformación de un ciclo expansivo del crédito financiado artificialmente con depósitos a la vista, lo cuales tuvieron un comportamiento simétrico entre 2011-4 y 2016-1; creciendo ambos por encima de su línea de tendencia y valor promedio en el lustro 2005-2017.

Todo ello desato un espiral inflacionaria para dicho lapso redujo el crédito y los depósitos a la vista en términos reales, mientras la disponibilidad financiera aumentó, producto de la contracción de la economía venezolana, porque precisamente los fenómenos inflacionarios dilapidan el ahorro voluntario (depósitos a plazo) y con ello la inversión real que permiten apalancar el aparato productivo nacional.

Dicho fenómeno inflacionario, en el caso venezolano, es de origen es monetario y en el estudio pudo constatarse la cointegración a largo plazo que existe entre VTINPC, VTM1 y VTBM; mientras que, por el lado de la expansión artificial del crédito, se constató la cointegración a largo plazo de VTCCNEA, VTBM y VTCCN. Siendo VT$\mathrm{BM}$ una variable endógena cointegradas con VTINPC y VTCCNEA, recae en el BCV la mayor responsabilidad por medio de la política monetaria a través las variaciones de la base monetaria o "dinero de alta potencia", del crecimiento de los precios y de la expansión artificial del crédito.

La política monetaria venezolana en el lapso considerado en este trabajo deja ver elementos diferenciados preocupantes al ser prácticamente iguales la oferta de dinero en sentido restringido M1 y en sentido amplio M2 por la inexistencia de los depósitos a plazo. Este fenómeno se acentuó después del proceso de reconversión monetaria en el año 2008 y no antes. Después de 2008, los depósitos a plazo comenzaron a ser inferiores a las captaciones del público del sistema bancario; mientras a partir de 2014-1 a raíz del inicio de la contracción progresiva del crédito hasta 2017-4 los depósitos a la vista también comienzan a ser superiores a M1 y M2.

Todo lo anterior puso de relieve que la expansión de la oferta monetaria restringida y eventualmente en sentido amplio, producto de políticas de transferencias del Estado venezolano junto a una política de crédito blando, mediante el control del tipo de interés, produjo del lado de la demanda una baja en la utilidad marginal del dinero que promovió un mayor consumo y del lado de la empresa un costo de capital que hacía posible la existencia de negocios que con tasas de interés relativamente más altas no serían factibles. El sostenimiento sectorial caracterizado por la expropiación de tierras productivas, compra de empresas, intervenciones y consecuentemente 
cierre de establecimientos productivos junto a una política monetaria expansiva, sin las debidas correcciones en materia fiscal y cambiaria, promovieron una aceleración de la inflación tal y como lo expusieron los autores clásicos, austriacos y neoclásicos abordados en este estudio.

\section{REFERENCIAS}

Anderson, D., Sweeney, D. y Willians, T. (2008). Métodos cuantitativos para los negocios. (9 ed.). México, D.F.: Thomson.

Azuero, S. (1990). Contratos bancarios. Su significación en América Latina. Bogotá, D.C.: Biblioteca Felaban (Federación Latinoamericana de Bancos).

Baptista, A. (2011). Bases cuantitativas de la economía venezolana. Caracas: Artesanogroup.

BCV. (2017). Boletín de Indicadores Semanales. Semana \#49 2017. Caracas: BCV. Recuperado de http://www.bcv.org.ve/publicaciones/boletin-de-indicadores-semanales-semana-49-2017

Blanchard, O. (2005). Macroeconomía. México, D.F.: Pearson Prentice Hall.

Braun, E. (2013). A French Predecessor of the Austrian Theory of the Business Cycle- Marcel Labordère on the American Crisis of 1907. Journal of Prices \& Markets, 1(1), 49-56.

Cantillón, R. (2002). Essay on the nature of Trade in General. Indianápolis: Liberty Fund, Inc.

Casas, J. (2017). Implicaciones de los acuerdos del Fondo Monetario Internacional sobre la pobreza en Colombia. Económicas CUC, 38(1), 9-36. http://dx.doi. org/10.17981/econcuc.38.1.01

Chiang, A. y Wainwright, K. (2006). Métodos fundamentales de economía matemática. (4 ed.). México, D.F.: McGraw-Hill.

De Soto, J. (2011). Dinero, crédito bancario y ciclos económicos. Madrid: Unión.

Friedman, M. y Meiselman, D. (1963). The Relative Stability of Monetary Velocity and the Investment Multiplier in the United States, 1898-1958. In: Commission on Money and Credit, Stabilization Policies (pp. 165-268). Englewood Cliff: Pretince Hall. Available from https://space.mit.edu/bitstream/handle/1721.1/48963/relativestabilit00ando.pdf?sequence=1\&isAllowed=y

Friedman, M. (2005). The Optimum Quantity of Money. Piscataway: Aldine Transaction Publishers.

Gómez, R. (2008). La Teoría Del Ciclo Económico De Friedrich Von Hayek: Causas Monetarias, Efectos Reales. Cuadernos de Economía, 27(48), 47-69. Disponible en https://revistas.unal.edu.co/index.php/ceconomia/article/view/1451

Henríquez, G., Rada, J. y Torrenegra, A. (2016). Medición de variables psicológicas, económicas y sociales para identificar factores ocultos de los emprendedores en Barranquilla. Económicas CUC, 37(1), 177-202. https://doi.org/10.17981/econcuc.37.1.2016.08

Hernández, G. H. (2011). La gestión empresarial, un enfoque del siglo XX, desde las teorías administrativas científica, funcional, burocrática y de relaciones humanas. Escenarios, 9(1), 38-51. Disponible en http://hdl.handle.net/11619/1628

Hernández, R., Fernández, C. y Baptista, P. (2010). Metodología de la investigación. (5 ed.). México, D.F.: McGraw-Hill. 
Herrera, A., Balbis, M., Gómez, A. y Hernández, H. (2015). Pragmatismo competitivo de las pymes de la Región Caribe. Barranquilla: CUL. Disponible en http:// hdl.handle.net/11323/3188

Jevons, S. (1998). La Teoría de la economía Política. Madrid: Pirámide.

Lorente, L. (2019). Crecimiento, crédito e inflación. Revista de Economía institucional, 21(40), 9-68. https://doi.org/10.18601/01245996.v21n40.02

Mises, L. (1982). The Theory of Money and Credit. Indianapolis: Liberty Fund.

Palella, S. y Martins, F. (2012). Metodología de la investigación cuantitativa. (5 ed.). Caracas: FEDUPEL.

Paredes, J. (2017). Planificación financiera ante la perspectiva organizacional en empresas cementeras del estado ZuliaVenezuela. Económicas CUC, 38(1), 105132. http:// dx.doi.org/10.17981/econcuc.38.1.05

SUDEBAN. (2017). Información Estadística. [Online]. Disponible en http://www.sudeban.gob.ve/index.php/gide_informacion-estadistica/

Thornton, H. (2000). Crédito papel. Madrid: Pirámide.

Urdaneta, A. (2017). Mecanismos de transmisión de la política monetaria en la economía venezolana 1984-2013. Revista Innovación y Gerencia, 9(1), 69-95.

Urdaneta, A., Prieto, R. y Hernández, O. (2017). Formación bruta de capital fijo en el producto interno bruto venezolano en el período 1997-2015. Desarrollo Gerencial, 9(1), 52-80. https://doi.org/10.17081/dege.9.1.2725

Villar, L., Salamanca, D. y Murcia, A. (2005). Crédito, represión financiera y flujos de capitales en Colombia: 1974-2003. Revista Desarrollo y Sociedad, (55), 167-209. https://doi.org/10.13043/dys.55.4

Wicksell, K. (1962). Interest and Prices. A study of the causes regulating the value of money. New York: Kelley.

Zúñiga, J. (2017). De la función económica del cheque, del cheque común al de pago diferido. Jurídicas CUC, 13(1), 183-198. https://doi.org/10.17981/juridcuc.13.1.2017.08

\section{BIODATA}

Remedios Pitre Redondo es Economista de la Universidad del Magdalena (Colombia). Magister Desarrollo y gestión de empresas de la Universidad Simón Bolívar (Colombia). Doctora en ciencias mención gerencia de la Universidad Rafael Belloso Chacín (Venezuela). Docente Universidad de la Guajira (Colombia). https://orcid.org/0000-0001-7373-1101

Armando Urdaneta Montiel es Doctor en Ciencias Económicas. Doctor en Ciencias Gerenciales de la Universidad Rafael Belloso Chacín. Magister en Telemática de la Universidad Rafael Belloso Chacín. Magister en Gerencia Empresarial de la Universidad Rafael Belloso Chacín. Ingeniero en Computación de la Universidad Rafael Belloso Chacín. Docente de la Universidad Metropolitana del Ecuador (Ecuador). https://orcid. org/0000-0002-9825-9453

Hugo Hernández Palma es Ingeniero Industrial de la Universidad Libre (Barranquilla, Colombia). Magister en sistema de gestión Universidad Autónoma del Caribe (Colombia). Docente Facultad Ciencia Económica Universidad del Atlántico (Colombia). https:/orcid. org/0000-0002-3873-0530 